\title{
Zur temporalen Entfaltung und multimodalen Orchestrierung von konzeptuellen Räumen am Beispiel einer Erzählung*
}

\author{
Silva H. Ladewig und Lena Hotze (Frankfurt (Oder))
}

\begin{abstract}
The study presented in this article investigates the temporal unfolding and multimodal orchestration of meaning in a narration. Two aspects are focused on. First, the temporal and multimodal orchestration of conceptual spaces in the entire narrative is described. Five conceptual spaces were identified which were construed by multiple visual-kinesic modalities and speech. Moreover, the study showed that the conceptual spaces are often created simultaneously, which, however, does not lead to communication problems due to the media properties of the modalities involved (see also Schmitt 2005). The second part of the analysis zoomed in onto the phase of the narrative climax in which the multimodal production of the narrative space with role shift dominated. By applying a timeline-annotation procedure for gestures (Müller/Ladewig 2013) a temporally unfolding salience structure (Müller/Tag 2010) could be reconstructed which highlights certain semantic aspects in the creation and flow of multimodal meaning. Thus, specific information "necessary" to understand the climax of the narration was foregrounded and made prominent for a co-participant. By focusing methodically and theoretically on the temporal structure and the interplay of different modalities, the paper offers a further contribution to the current discussion about temporality, dynamics and multimodality of language (Deppermann/Günthner 2015; Müller 2008b).
\end{abstract}

\section{$1 \quad$ Einleitung}

Erzählen ist eine kommunikative, rekonstruktive und koordinierte Aktivität der Gesprächsteilnehmenden. Indem eigene Erlebnisse oder Fremderfahrungen erzählt werden, können Sprecher*innen unter anderem einen Sachverhalt illustrieren, Vergangenes strukturieren und sequenzieren oder über jemanden klatschen (cf. Quasthoff 2001: 1295). Der (autobiographischen) Erzählung selbst kommt dabei oft eine soziale Funktion zu, da nicht nur die dargestellten Erlebnisse rekonstruiert und re-interpretiert werden (Schütze 1994), sondern auch, weil „Facetten von Identität“" sowohl hergestellt als auch dargestellt werden (Lucius-Hoene/Deppermann 2002: 63). Erzählen ist damit eine im Alltag fest integrierte Praktik (cf. Gülich 2008), deren Prozessualität und Interaktivität gerade in interaktionalen Ansätzen herausgestellt und erforscht worden ist.

\footnotetext{
* Wir bedanken uns bei den Gutachter*innen für ihre äußerst konstruktive Kritik und bei @Mathias Roloff (mathiasroloff.de) für die Zeichnung, die für Abbildung 3 und 6 verwendet wurde.
} 
Obwohl die Erzählung als „big package“ (Sacks 1992b: 354) beschrieben wurde und somit gut als Gestalt von Interaktionspartner*innen erkennbar ist, plädiert u. a. Gülich (2007: 38) dafür, Erzählungen nicht als abgegrenzte, durch Erzählende allein konstruierte Einheit zu behandeln, sondern „Erzählen als koordinierte Aktivität aller Beteiligten, die sich aus dem Gesprächsprozess heraus entwickelt“ zu betrachten. Erzählen ist demnach als „gemeinsame Leistung“ (Gülich 2005: 74) zu verstehen, das heißt als interaffektiven, kognitiven und interaktiven Prozess. Dabei bilden affektive Erfahrungen die Grundlage von Intersubjektivität (cf. Horst 2018; Merleau-Ponty 1962/2005; Müller/Kappelhoff 2018) im Prozess des Erzählens. Affektivität ist dynamisch und interaktiv. Sie emergiert lokal als geteilte Erfahrung ("Interaffektivität", Fuchs 2017; Horst et al. 2014) von Ko-Partizipant*innen und entfaltet sich im Verlauf einer Interaktion.

It is such a continuous inter-affective interaction of the participants that creates the temporal development of affect over time and grounds the course of the conversation. The dynamic pattern of jointly created affect goes along with the negotiation of a shared attitude towards a given topic.

(Horst et al. 2014: 2122)

Intersubjektivität ist folglich affektiv grundiert, denn sie basiert auf einem kollektiven „SichGewahrwerden" und einem gemeinsamen Erleben in einer sich temporal entfaltenden Gesprächssituation.

The intersubjectivity of the "we-relationship" in face-to-face interaction is rooted in temporality: It rests on the possibility that subjects mutually synchronize their consciousness by perceiving one another in shared time [...] from the point of view of the ever-moving, fleeting present.

(Deppermann/Günthner 2015: 3)

Wie Affektivität ist auch semantische und pragmatische Bedeutung als dynamisch aufzufassen, denn diese ist nicht statisch an einzelne Wörter gebunden, sondern emergiert lokal in einer Interaktion und wird interaktiv ausgehandelt (cf. u. a. Cameron 2002; Deppermann 1999; Kallmeyer 1981; Müller 2008b). Bedeutung ist ferner kognitiv und wird von den Partizipant*innen einer Interaktionssituation ko-konstruiert. Sie ist folglich als ein „doing“ (Gibbs 2018; Jensen 2017; Müller/Kappelhoff 2018) zu verstehen, d. h. als ein Kreieren und Etablieren von Bedeutung im Verlauf einer Interaktion. Sprich, die Konstruktion mentaler Räume (Dancygier 2011; Ehmer 2011; Fauconnier 1985; Fauconnier/Sweetser 1996), das mit dem Erzählen verbundene Erinnern (cf. Lucius-Hoene/Deppermann 2002; Quasthoff 1980) oder Imaginieren (cf. u. a. Ehmer 2011) sind demnach als kooperatives Herstellen von Bedeutung zu verstehen.

Eine Voraussetzung für das gemeinsame Erzeugen von Bedeutung ist die geteilte Aufmerksamkeitsausrichtung, für die das Zuhören wesentlich ist (cf. Gülich 2005: 75). Im Hinblick auf die Verfasstheit von Face-to-Face Interaktion ist jedoch nicht nur das Zuhören, sondern auch das Zusehen zentral. So schließt Müller (2003: 263) beispielsweise Gesten in die Analyse narrativer Strukturen mit ein:

The gestures [...] are part of the narrative structure of a verbally and bodily described event. They create a visual display of the narrative structure and thus turn the story telling into a multi-modal event: something to listen to but also something to watch [...] they are natural elements of an everyday rhetoric of telling a story in conversation.

(Müller 2003: 263) 
Auf die multimodale Verfasstheit des Sprechens hat die moderne Gestenforschung bereits in den 1970er Jahren hingewiesen (cf. u. a. Kendon 1972). Seit den 1990er Jahren wurden Gesten verstärkt in die Analyse von Narrationen einbezogen. So erarbeiteten Cassell/McNeill (1991) eine Typologie von Gesten, wie sie im Kontext von Erzählungen zu finden ist und zeigen, dass Sprecher*innen den Gebrauch verschiedener Gestentypen je nach Erzählebene variieren. Die metanarrative Ebene zeichnet sich beispielsweise durch ein hohes Vorkommen an deiktischen und metaphorischen Gesten aus, während die narrative Ebene einen verstärkten Gebrauch von ikonischen bzw. konkret referierenden Gesten aufweist. Diese letztgenannten Gesten, die konkrete Handlungen und/oder Objekte verkörpern, ermöglichen es auch, die sprecherseitige Perspektive auf einen Redegegenstand zu rekonstruieren. Werden beispielsweise Gesten im Modus des Agierens ausgeführt und Handlungen nachgeahmt (cf. Müller 1998, 2014), so wird aus der Perspektive von Aktant*innen erzählt. Ereignisse werden folglich so konstruiert und präsentiert, wie sie körperlich erfahren werden, weswegen sie als „,character viewpoint gestures“ bezeichnet wurden (McNeill 1992; Tuite 1993 ). Ferner können Sprecher*innen aus der Beobachter*innenperspektive erzählen, die in so genannten „observer view-point gestures“ (McNeill 1992; Tuite 1993) sichtbar wird. Diese Gesten werden im Modus des Repräsentierens durchgeführt (cf. Müller 1998; Müller 2014). Hierbei wird die Hand zum Objekt, wobei die Bewegung der Hand die Bewegung dieses Objektes verkörpert. Ein*e Sprecher*in kann demgemäß ein und dasselbe Ereignis aus unterschiedlichen Perspektiven gestisch verkörpern.

Seit der von McNeill vorgenommen Differenzierung ikonischer Gesten in viewpoint gestures haben sich diese zu einem stark beforschten Untersuchungsfeld entwickelt und damit einhergehend auch multimodale Erzählungen. So zeigten Studien beispielsweise, dass der Gebrauch von viewpoint gestures“ mit bestimmten syntaktischen Konstruktionen korreliert. „Character viewpoint gestures“ kommen demnach eher mit transitiven Verben und einfachen Sätzen vor, wohingegen ,observer viewpoint gestures“ mit intransitiven und komplexen Sätzen ko-okkurieren (cf. Beattie/Shovelton 2001, 2002; McNeill 1992; Parrill 2010). Ferner zeigten Forscher*innen, dass „,character viewpoint gestures“ häufig eine artikulatorische Dichte und damit eine höhere semiotische Komplexität aufweisen (cf. Bressem/Ladewig/Müller 2018; Brown 2008; Casey/Emmorey 2009; Duncan 2005; Quinto-Pozos/Parrill 2015). Werden mehrere Artikulatoren genutzt, wird die sprachlich-gestische Darstellung nicht nur semantisch dichter, sondern es können mehrere Perspektiven (,,viewpoints“) miteinander verbunden werden, ähnlich wie es bei Gebärdensprachen zu beobachten ist (cf. Cormier et al. 2012; Quinto-Pozos/Parrill 2015). Dies bedeutet konkret, dass mehrere Perspektiven in einer gestischen Darstellung verkörpert werden können (cf. Earis/Cormier 2013; Frederiksen 2017; Parrill 2009), bei denen der Körper gewissermaßen partitioniert wird (cf. Dudis 2004). Ein Wechsel im Gebrauch einzelner Artikulatoren kann demnach mit einem Wechsel der Perspektive korrelieren, der nicht immer sprachlich angezeigt werden muss. So konnten Maury-Rouan (2011) und McClave (2000) zeigen, dass ein Perspektivwechsel wie bei Rollenübernahmen oder Einschüben von Kopfbewegungen zur Seite und der Abwendung des Blickes des*der Sprechers*in von Ko-Partizipant*innen begleitet werden kann (für weitere Beispiele siehe die umfangreiche Aufstellung von (cf. Stec 2012) für gesprochene und gebärdete Sprachen). Damit einher geht die Aufteilung des Gestenraumes in einzelne konzeptuelle Räume. So ist der real existierende Raum, in dem sich Interaktionsteilnehmende befinden, Gegenstand individueller aber auch gemeinsamer Konzeptualisierung der Interaktant*innen (cf. Croft/Cruse 2004; Fauconnier 1985; Ehmer 2011: 34- 
35). Gleichsam konstruieren Gesprächsteilnehmende „interaktionale Zonen“ (Sweetser/Stec 2016: 244), narrative Räume (,Story Spaces“, Sweetser/Stec 2016), die besonders für Rollenübernahmen genutzt werden („character enactment“, bei Goffmann 1981 auch „footing“,) oder Gedächtnisräume („Memory Space“, Sweetser/Stec 2016: 253), die zum Überlegen und zur Wortsuche genutzt werden.

Die funktionale Partitionierung des Körpers (cf. Dudis 2004; Haddington et al. 2014; Schegloff 1998) erlaubt es, dass diese mit verschiedenen Perspektiven korrelierenden Räume simultan konstruiert werden können. Oft ist es der Blick einer*s Sprechers*in, der diese Überschneidung ermöglicht. So kann sprachlich und gestisch ein narrativer Raum kreiert, jedoch zeitgleich mittels Blickverhaltens eine interaktionale Achse zwischen den Gesprächspartner*innen etabliert werden, um beispielsweise die Aufmerksamkeit zu steuern oder Ko-Partizipant*innen als Mitspielende zu rekrutieren (cf. Thompson/Suzuki 2014).

Die unterschiedlichen Perspektiven auf einen Redegegenstand, die in verschiedenen Modalitäten sichtbar werden können, korrelieren auch mit unterschiedlichen Diskursstatus von Informationen und narrativen Strukturen. „Character viewpoint gestures“ werden demnach gebraucht, um zentrale Informationen zu vermitteln und um Affekte zu verkörpern. „Observer viewpoint gestures“ hingegen werden stärker genutzt, wenn periphere Informationen kommuniziert werden (McNeill 1992: 120-125; cf. auch Kita/Özyürek 2003; Parrill 2012). Zudem beschreibt McNeill (1992: 125-128), dass Gesten die Relevanz und Salienz von Informationen verdeutlichen. So zeigt die gestische Form, auf welche semantischen Facetten Sprecher*innen ihre Aufmerksamkeit lenken. Dieser Aspekt ist im Ansatz der dynamischen, multimodalen Kommunikation theoretisch und methodisch ausgearbeitet worden. Gegenstand der Analysen sind die Emergenz, Entfaltung und interaktive Aushandlung von multimodaler Bedeutung über längere Zeiträume hinweg. Bedeutung wird dabei nicht als Produkt, sondern als Prozess verstanden (Cameron 2002; Müller 2008a), bei dem über den Gebrauch verschiedener Modalitäten einzelne Bedeutungsaspekte hervorgehoben und salient gesetzt werden können (cf. u. a. Kappelhoff/Müller 2011; Kolter et al. 2012; Müller 2007, 2008a, b, 2017; Müller/Ladewig 2013; Müller/Tag 2010). Die dabei entstehende multimodale Salienzstruktur macht wandernde Aufmerksamkeitsfoki von Sprecher*innen (Chafe 1994) sichtbar. Diese können wiederum zum Gegenstand interaktiver Aushandlungsprozesse werden und so die Aufmerksamkeit von KoPartizipanten*innen lenken. Mit dem analytischen Blick auf alle Ko-Partizipant*innen einer Interaktionssituation verbindet sich dieser anfänglich kognitiv-linguistische Ansatz auf Bedeutungskonstitution, der sich in den letzten Jahren zu einem phänomenologischen Ansatz entwickelt hat (Müller/Kappelhoff 2018), mit einer interaktiven Perspektive auf Bedeutungsaushandlung, die Aufmerksamkeit als gemeinsame Aktivität der Interaktionspartner*innen versteht (cf. Goodwin 1981; cf. dazu Müller 2017).

Der theoretische Rahmen der dynamischen multimodalen Kommunikation, der sich bisher auf den Gegenstand der Metaphern in verschiedenen Diskurskontexten sowie unterschiedlichen audio-visuellen Medien (beispielsweise Film) fokussierte, bildet den Rahmen für die folgende qualitative Untersuchung. Dabei werden wir uns auf konkrete Bedeutung (in Abgrenzung zu abstrakter Bedeutung) konzentrieren, wie sie im Kontext einer Erzählung multimodal, d. h. sprachlich und gestisch, orchestriert und elaboriert wird. Ziel ist es, die temporale Entfaltung 
und multimodale Orchestrierung konzeptueller Räume zu untersuchen. Dazu werden wir im nächsten Schritt unser methodisches Vorgehen einführen.

\section{Methodisches Vorgehen}

Die qualitative Analyse ${ }^{1}$ unseres Beispiels erfolgte in drei Schritten, die sich durch verschiedene „levels of granularity“ (Schegloff 2000: 719) auszeichnen.

Diese sind:

1. Annotation von Geste und Rede nach LASG (Bressem/Ladewig/Müller 2013) in Elan (Wittenburg et al. 2006),

2. (Metaphor) Foregrounding-Analyse in Keynote (MFA, Müller/Tag 2010) und

3. Zeitstrahlannotation in Keynote (KeaTA, Kolter et al. 2012; Müller/Ladewig 2013).

Die Analyse von Rede und Geste folgte dem linguistischen Ansatz auf Gesten, der von der Beobachtung ausgeht, dass Gesten alle drei Sprachfunktionen nach Bühler (1934) erfüllen können und somit ein sprachliches Potenzial aufweisen (cf. Müller 2013), was ihre Entwicklung hin zu Gebärdensprachen belegt (cf. u. a. Pfau/Steinbach 2006; Wilcox 2004). Diese theoretischen Annahmen korrelieren mit einem methodischen Ansatz, der die Motivation einer Geste und damit die Analyse ihrer Form ins Zentrum stellt. Gesten werden demgemäß zunächst unabhängig von der Sprache im Hinblick auf Formparameter, gestische Darstellungsweisen, Schemata und motorische Muster untersucht. Im zweiten Schritt erfolgt die feinkörnige Analyse des Zusammenspiels von Rede und Geste auf den Ebenen der Semantik, Syntax und Pragmatik (für weitere Informationen cf. Bressem/Ladewig/Müller 2013; Müller/ Bressem/Ladewig erscheint).

\begin{tabular}{|c|c|c|}
\hline keine Hervorhebung & $\begin{array}{l}\text { Hervorhebungsstrategien } \\
\text {,foregrounding strategies' }\end{array}$ & $\begin{array}{c}\text { Hervorhebung } \\
\text { von Bedeutungsaspekten durch }\end{array}$ \\
\hline $\begin{array}{l}\text { monomodales } \\
\text { Konstruieren von } \\
\text { Bedeutung }\end{array}$ & $\begin{array}{c}\text { Ikonizitätsprinzip } \\
\text { und/ oder } \\
\text { Interaktives Prinzip } \\
\text { und/ oder } \\
\text { Semantisches \& } \\
\text { syntaktisches Prinzip }\end{array}$ & $\begin{array}{l}\text { multimodales Konstruieren von } \\
\text { Bedeutung (simultan) oder } \\
\text { Wiederholung von monomodalen oder } \\
\text { multimodalen Einheiten } \\
\text { Blickrichtung (auf Objekt oder Gesten), } \\
\text { größere Bewegungen, Prosodie } \\
\text { Gesten füllen syntaktische } \\
\text { und semantische Lücke }\end{array}$ \\
\hline
\end{tabular}

Abbildung 1: Strategien der Hervorhebung von Bedeutung nach Müller/Tag (2010)

\footnotetext{
${ }^{1}$ Verschiedene Schritte der Annotation werden hier unter „Analyse“ gefasst, da sowohl die Bestimmung von Einheiten als auch ihre Beschreibung Ergebnisse analytischer Schritte sind, die wesentlich vom theoretischen Bezugsrahmen beeinflusst werden.
} 
Im zweiten Analyseschritt werden die von den Gesprächsteilnehmenden angewandten ,Hervorhebungsstrategien“ (,foregrounding strategies“, Müller/Tag 2010) annotiert, die das Salientsetzen einzelner Bedeutungsaspekte sichtbar machen (siehe Abbildung 1). Die verschiedenen Strategien werden nach Müller/Tag (2010) unter dem „Ikonizitätsprinzip“, dem „Interaktiven Prinzip“ sowie dem „Semantischen und Syntaktischen Prinzip“ zusammengefasst und wie folgt verstanden: Werden Bedeutungsaspekte in mehr als einer Modalität ausgedrückt (simultan oder in kurzer linearer Abfolge), so sind diese hervorgehoben. Diese Strategie folgt dem Ikonizitätsprinzip, wonach mehr Material mehr Bedeutung ausdrückt (cf. Jakobson 1966; Mayerthaler 1980; Müller/Tag 2010; Pörings/Schmitz 2003). Zudem kann der Gebrauch von Salienzmarkierungen Bedeutung hervorheben. Hierzu zählen u. a. die Blickrichtung, um gemeinsame Aufmerksamkeitsausrichtung zu initiieren (cf. u. a. Gullberg/Kita 2009; Streeck 1988, 2009) oder das Betonen von sprachlichen Einheiten (Interaktives Prinzip). Andere Modalitäten können durch eine semantische und syntaktische Integration in die Äußerung mittels deiktischer Partikel (Fricke 2012) oder mittels Substitution lautsprachlicher Einheiten (Ladewig 2014a, 2020) hervorgehoben werden. ${ }^{2}$

Wie Abbildung $1 \mathrm{zu}$ entnehmen ist, können diese Prinzipien einzeln oder simultan auftreten.

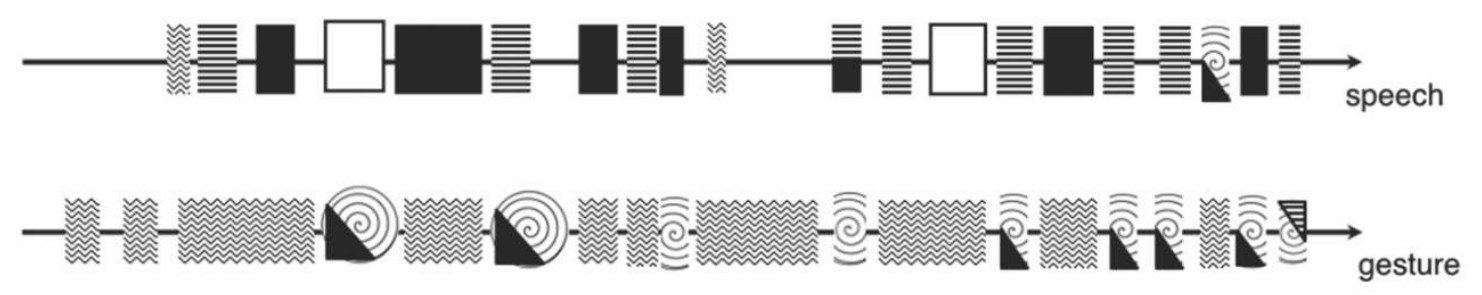

spiral

wave-like movement

downward

upward

$\square$ no metaphoricity

Abbildung 2: Zeitstrahlannotation in Keynote (Müller/Ladewig 2013: 305)

Stehen in den ersten beiden Schritten das feingliedrige Zusammenspiel von Rede und Geste im Vordergrund, wird im dritten Schritt der Analysefokus auf die Diskursebene erweitert und die temporale Entfaltung sprachlich-gestischer Bedeutung in den Blick genommen, die mit Hilfe der Keynote basierten Zeitstrahl-Annotation („Keynote based Timeline Annotation“, KeaTA) erfasst wird. Dieses System wurde ursprünglich entwickelt, um multimodale Metaphern und Ausdrucksbewegung in audio-visuellen Medien zu untersuchen (cf. Kappelhoff/Müller 2011; Müller/Kappelhoff 2018; Müller/Schmitt 2015; Schmitt/Greifenstein/Kappelhoff 2014) und später hinsichtlich der Analyse sprachlich-gestischer Metaphern adaptiert (Kolter et al. 2012; Müller/Ladewig 2013). Abbildung 2 zeigt eine Teildarstellung einer solchen Annotation, die den repetitiven Gebrauch verschiedener Metaphern über einen längeren Zeitraum einer therapeutischen Sitzung visualisiert. Die grafischen Muster dieser Zellen entsprechen wesentlich der Bedeutung, wie sie in Geste und Lautsprache vermittelt werden. Die Größe der Muster bildet grob die Länge der identifizierten monomodalen und multimodalen Äußerungen ab. So stellen

\footnotetext{
${ }^{2}$ Für eine genaue Beschreibung der einzelnen Schritte cf. Müller/Tag (2010: 97).
} 
die in Abbildung 2 gezeigten nach oben und nach unten gerichteten Pfeile die sprecherinnenseitige Konzeptualisierung des Lebens als ein Auf und Ab dar. Vergleicht man die Vorkommen sprachlicher und gestischer metaphorischer Ausdrücke (oberer Zeitstrahl und unterer Zeitstrahl in Abbildung 2) so fällt auf, dass sowohl die Auf- als auch die Abwärtsbewegung des Lebens lautsprachlich konstruiert werden. Gestisch jedoch wird häufig eine Abwärtsbewegung verkörpert (zum sprachlichen und gestischen Ausdruck von Metaphern cf. Cienki/Müller (2014)). Umgekehrt verhält es sich mit den anderen Metaphern, mithilfe derer die Patientin über ihr Leben spricht, wie die der Welle (Wellenform in Abbildung 2) oder der Spirale (Spiralform in Abbildung 2). Beide können sich mit der erst genannten Metapher verbinden, werden jedoch nur an bestimmten Punkten der Therapiesitzung verbalisiert und größtenteils gestisch ausgedrückt. Mithilfe dieser Methode und der daraus resultierenden Ergebnisse konnten Kolter et al. (2012) u. a. Rückschlüsse über den Therapiefortschritt der untersuchten Patientin ziehen.

Die folgenden Abschnitte stellen die Analyse einer Erzählung vor, deren Ziel es war, die temporale Entfaltung und multimodale Orchestrierung konzeptueller Räume zu untersuchen. Wie in der Analyse selbst wandert der Analysefokus in der Vorstellung der Studie von der Makroebene und der darauf untersuchten multimodalen Erzeugung und temporalen Entwicklung konzeptueller Räume zur Mikroebene, auf der das Zusammenspiel einzelner Modalitäten in der Konstruktion von Bedeutung untersucht wurde. Theoretischer und methodischer Hintergrund ist der Ansatz der dynamischen und multimodalen Kommunikation, welcher eben skizziert wurde.

\section{Erzählung „Oma ich hab doch ’n Schlüssel vergessen““}

Die untersuchte Erzählung ist einem Videokorpus entnommen, das zur Analyse von sprachlich unterbrochenen und gestisch vervollständigten Äußerungen erstellt wurde (cf. Ladewig 2014a, 2019, 2020). Das Korpus basiert auf 20h Videomaterial verschiedener Diskurstypen, hauptsächlich Alltagsgesprächen, aber auch Gesellschaftsspielen und Talkshows. Die Daten wurden im Zeitraum von 2004-2010 erhoben. Die hier fokussierte Erzählung entstammt aus einer 2005 dokumentierten Unterhaltung - einem geselligen Beisammensein bei Kaffee und Kuchen zwischen vier weiblichen Mitgliedern einer Familie, im Alter zwischen 30 und 80 Jahren. Wir konzentrieren uns in der Nacherzählung sowie in der sich anschließenden Analyse nur auf die im Datenmaterial identifizierten monomodalen und multimodalen Äußerungen. Kontextuelle Informationen, die allein den Interaktionsteilnehmerinnen zur Verfügung standen, können aufgrund von Unkenntnis nicht miteinbezogen werden, weswegen die Erzählung selektiv unverständlich erscheinen könnte („Indexikaliät“, cf. Garfinkel 1967). Im Folgenden soll die Erzählung kurz wiedergegeben werden, soweit es das Medium Schrift erlaubt. Detaillierte Informationen zu den verbal-gestischen Äußerungen finden sich in den nächsten Abschnitten. Ein Transkript kann im Anhang eingesehen werden.

Gegenstand der Erzählung ist ein Erlebnis, das sich am Hochzeitstag der Sprecherin, genauer gesagt während der Heimkehr von ihrer Hochzeitsfeier, ereignet hat. Dabei gehört die Sprecherin zu einer der vier Charaktere: die Braut (die Sprecherin), der Bräutigam, die Schwester und die Oma der Braut. Die Erzählung beginnt mit der Rekonstruktion der Heimfahrt bis zur Wohnung der Schwester, in der die Oma die Tochter des Brautpaares betreut. Dort angelangt, muss die Schwester feststellen, dass sie den Schlüssel zu ihrer Wohnung vergessen hat. Um in ihre 
Wohnung zu gelangen, versucht sie über das Fenster in die Wohnung zu klettern. Ihre Oma jedoch, die am Fenster in der Wohnung steht, bemüht sich, diese Unternehmung zu verhindern. An diesem Punkt der Erzählung beginnt die Sprecherin, mehrere Rollenwechsel zu vollziehen. Zunächst kündigt sie mit der Äußerung und Oma imma einen Rollenwechsel von der Erzählerin zu einer der Protagonistinnen, ihrer Oma, an (,,projection“, Sacks/Schegloff/Jefferson 1974), den sie sodann ausführt und ihre Oma sprachlich und gestisch imitiert. Genauer gesagt, reproduziert sie die Äußerungen der Oma was WILLst DENN DU? GEH DU rUNta in stimmlicher Qualität, Intonation, Dialekt sowie Lautstärke und führt dabei zwei Gesten aus, die die Handlung des Schubsens verkörpern (eine Visualisierung des temporalen Verlaufs sprachlich-gestischer Äußerungen findet sich in Abbildung 5 und Abbildung 7). Im Anschluss an diese Verhaltensimitation wechselt die Sprecherin in die Rolle der Erzählerin und gibt weitere Kontextinformationen, indem sie Fenster- und Wohnungstyp mit der Äußerung das war ne zweiraumwohnung hier mit so 'nem Gitta präzisiert und auf eine Wohnung des gegenüberliegenden Wohnhauses zeigt. Sodann begibt sich die Sprecherin zurück in die Erzählung und rekonstruiert den weiteren Verlauf des Geschehens. Sie kündigt einen zweiten Rollenwechsel zur Schwester an (und hanne imma) und imitiert anschließend ihr sprachliches und körperliches Verhalten (oma ich hab doch $n$ schlüssel vergessen plus beidhändige nach oben gerichtete Hand). Die darauffolgende Reaktion ihrer Oma wird mit einer weiteren Rollenübernahme verkörpert (geh Runta plus Geste, die die Handlung des Nach-Unten-Schubsens darstellt). Im Anschluss daran fasst die Sprecherin sprachlich das weitere Geschehen zusammen. Demnach versuchte sie, die Schwester nach oben zu drücken, wobei ihre Oma dem entgegenwirkte, indem sie sie nach unten drückte. Auf der gestischen Ebene werden die Handlungen des Nach-Unten- und NachOben-Drückens verkörpert. Währenddessen lachen die weiteren Ko-Partizipanten*innen. Die Sprecherin kommentiert die Erzählung am Ende mit der Äußerung es war so witzig und markiert so die Würdigkeit der Erzählung (,reportability“, Gülich/Hausendorf 2000; Hausendorf/Quasthoff 1996) mit Hilfe einer Evaluation (cf. Labov 1972).

Der folgende Abschnitt rekonstruiert die temporale Entfaltung und multimodale Konstruktion von konzeptuellen Räumen über die gesamte Erzählung hinweg. Die mikroanalytische Untersuchung des Zusammenspiels der einzelnen Modalitäten wird in dem darauffolgenden Abschnitt (3.2) vorgestellt.

\subsection{Multimodale Etablierung und temporale Entfaltung konzeptueller Räume}

Multimodale Erzählungen sind äußerst vielschichtig und komplex, ${ }^{3}$ was wir im Folgenden anhand der multimodalen Konstruktion und temporalen Entfaltung von Erzählräumen illustrieren. Dabei gehen wir der Mental Space Theory folgend (Fauconnier 1985; Fauconnier/Turner 2002) davon aus, dass im Verlauf eines Diskurses unterschiedliche konzeptuelle Räume eröffnet und etabliert werden können, die hierarchische Strukturen zeigen. Wie zahlreiche Studien zu Erzählungen bei Erwachsenen und Kindern gezeigt haben, können diese im real-existierenden Raum verankert werden (cf. Ehmer 2011; Heller 2018; Sweetser/Sizemore 2008; Sweetser/Stec 2016). So kann der zwischen zwei Gesprächspartner*innen geteilte Raum funktional so aufgeteilt werden, dass beispielsweise die Achse zwischen Sprecher*in und Gesprächspartner*in einen interaktionalen Raum etabliert und der angrenzende Raum funktional als narrativer Raum

\footnotetext{
${ }^{3}$ Cf. dazu auch die Beiträge in (König/Oloff 2018).
} 
konstruiert wird (cf. Sweetser/Sizemore 2008; Sweetser/Stec 2016). In einem narrativen Raum wiederum kann eine einzige gestische Darstellung weitere konzeptuelle Räume eröffnen und physisch verankern (cf. Sweetser 2013). ${ }^{4}$

Die Konstruktion und physische Verankerung konzeptueller Räume kann auch in der hier untersuchten Erzählung beobachtet werden. Auf Basis des „Reality Space“, den wir nach Ehmer (2011: 35) als die „,von den Sprechern geteilte mentale Repräsentation der aktuellen Kommunikationssituation“ verstehen, eröffnet die Sprecherin sprachlich, gestisch und mittels Blicks fünf konzeptuelle Räume: einen interaktionalen Raum (blaue Färbung in Abbildung 3) einen narrativen Raum (orange Färbung in Abbildung 3), einen metanarrativen Raum (grüne Färbung in Abbildung 3), einen Reflexionsraum (rote Färbung in Abbildung 3), der sowohl Überlegungen als auch Wort- und Formulierungssuchen einschließt, und einen neutralen Raum (graue Färbung in Abbildung 3), in dem keine kommunikative Aktivität zu verzeichnen ist (beispielsweise, wenn die sich die Hände in einer Ruheposition ${ }^{5}$ befinden oder der Blick beim Luftholen von Ko-Partizipant*innen abgewendet wird). Diese Räume wurden auf Basis folgender, aus dem Material heraus entwickelten Merkmale bestimmt: Die Rekonstruktion sprachlich konstruierter Räume erfolgte anhand semantischer, pragmatischer und prosodischer Merkmale, wie u. a. Intonation, Betonung und Lautstärke. So wies beispielsweise die sprecherinnenseitige Nachahmung stimmlicher Qualitäten einer Protagonistin der Erzählung (,enactment“) auf die Etablierung des Erzählraumes hin. Lieferte die Sprecherin Hintergrundinformationen oder evaluierte sie das Erzählte, so wurde dies als Mittel der Herstellung des metanarrativen Raumes interpretiert. Eine direkte Ansprache der Interaktionspartnerinnen wies auf die Herstellung eines interaktionalen Raumes hin. Der Gebrauch von Häsitationsmarkierungen wurde als Mittel zu Konstruktion des Reflexionsraumes interpretiert. Längere Pausen, die keine Anzeichen von Reflexion zeigten, wurden als Mittel zur Konstruktion des neutralen Raumes gewertet.

Die Raumkonstruktion auf der Ebene des Blickverhaltens wurde anhand der Blickrichtung und der damit einhergehenden Kopfbewegungen rekonstruiert. So etablierte die Sprecherin beispielsweise eine Achse zu ihren Gesprächspartnerinnen, indem sie sie ansah. Der nach vorn gerichtete Blick wurde entweder als neutraler Raum oder Erzählraum rekonstruiert. Der diagonal nach links gerichtete Blick etablierte einen Reflexionsraum, hingegen der diagonal nach rechts gerichtete Blick einen metanarrativen Raum. Für die Bestimmung dieser Räume spielten weitere Merkmale wie sprachliche und/oder gestische Mittel eine Rolle (u. a. Häsitationen, Pausen, gestische Haltepositionen, Gestentypen). Ferner zeigte sich, dass Objekte oder Gesten mittels Blicks interaktiv relevant gesetzt wurden und so die Aufmerksamkeit aller Partizipantinnen auf diese Objekte oder Gesten gelenkt wurde (cf. dazu u. a. Gullberg/Kita 2009; Müller/Tag 2010; Streeck 1988, 2009). In diesem Fall wurde ein interaktionaler Raum eröffnet und ein Reflexionsraum etabliert, sofern die Sprecherin eine Formulierung oder ein Konzept suchte.

\footnotetext{
${ }^{4}$ Dass Gesprächspartner*innen sensibel für die gestische Aufteilung von Räumen sind und diese nicht nur erkennen sondern auch dort erwarten, wo sie etabliert worden sind, konnten Gunter/Weinbrenner/Holle (2015) anhand von Experimenten zeigen.

${ }^{5}$ Selbstverständlich können auch Ruhepositionen oder Atmen kommunikativ relevant sein (cf. u. a. FogartyBourget/Artemeva/Fox 2019)
} 


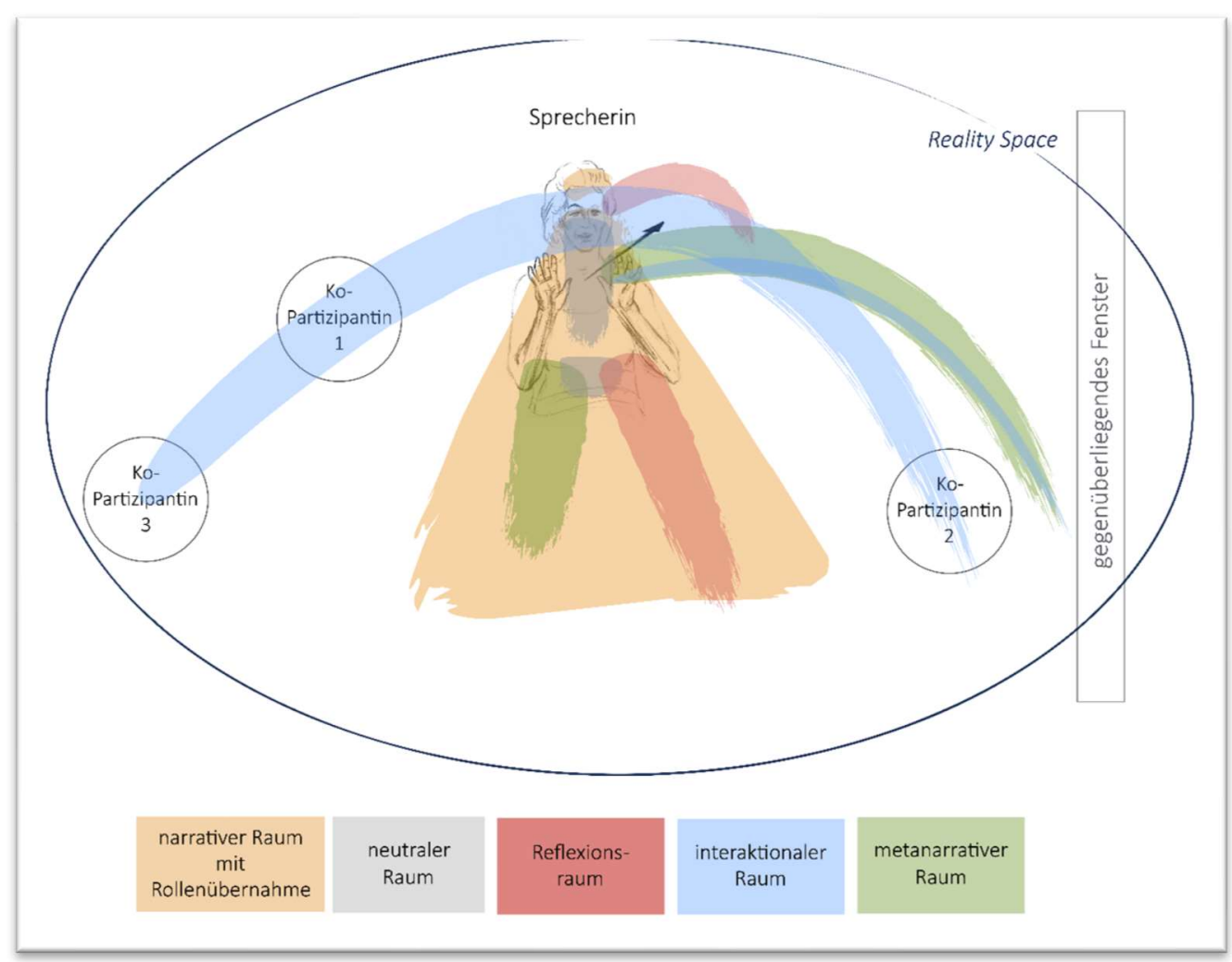

Abbildung 3: Erzählsituation und etablierte konzeptuelle Räume

Die gestische Etablierung von konzeptuellen Räumen wurde sowohl anhand von Gestentypen als auch der Bewegungsrichtung rekonstruiert. So wurden deiktische Gesten eher zur Übermittlung metanarrativer Informationen, wie beispielsweise Hintergrundinformationen, eingesetzt, wie Cassell/McNeill (1991) bereits beschrieben haben. Aber auch pragmatische Gesten (Kendon 1995; Payrató/Teßendorf 2014) bzw. rekurrente Gesten (Ladewig 2014b) wurden dazu genutzt, um metanarrative Räume zu eröffnen. Insbesondere der Gebrauch der nach oben gerichteten, flachen Hand (,,palm up open hand“, Müller 2004) konnte als gestisches Mittel zur Etablierung des metanarrativen Raumes identifiziert werden. Referentielle bzw. ikonische Gesten eröffneten einen narrativen Raum, wobei hier ausschließlich agierende Gesten zur Rollenübernahme identifiziert wurden. Hinsichtlich der Bewegungsrichtung stellte sich heraus, dass der vor der Sprecherin liegenden Gestenraum sowohl einen narrativen Raum als auch einen metanarrativen Raum eröffnete. Gesten, die in die Richtung der Ko-Partizipantinnen bewegt wurden, erfüllten immer eine interaktive Funktion und etablierten somit einen interaktionalen Raum. Diese korrelierten in der Regel mit dem Blick zu einer der Ko-Partizipantinnen. Der neutrale Raum wurde mittels Ruheposition am Körper der Sprecherin konstituiert.

Es sei darauf hingewiesen, dass die Rekonstruktion der einzelnen Räume im Zusammenspiel der hier adressierten Modalitäten und aus dem Prozess der Bedeutungsherstellung heraus erfolgte. Das heißt die hier verwendeten Annotationskategorien stehen nicht in einem absoluten Verhältnis zu den identifizierten Räumen. So kann eine gestische Bewegung, die nach links, in Richtung einer Ko-Partizipantin ikonisch die Bewegung einer Person nach links abbilden und somit einen narrativen Raum oder aber einen interaktionalen Raum eröffnen. Ebenso ist eine agierende Geste nicht gleichzusetzen mit der Rollenübernahme von Protagonist*innen, sondern kann auch Hintergrundinformationen liefern. 
Abbildung 3 stellt die anhand räumlich-visueller Modalitäten (Gesten, Blickverhalten inkl. Kopfbewegungen) bestimmten Räume dar, wonach die Sprecherin mittels Blickverhaltens interaktive Räume zu ihren Gesprächspartnerinnen etabliert, die links und rechts von der Sprecherin sitzen. Indem sie ihren Blick auf ein gegenüberliegendes Fenster richtet oder auf ihre Gesten, die sie über ihrem Kopf platziert, lenkt sie ihre Aufmerksamkeit und die ihrer Gesprächspartnerinnen auf dieses Objekt oder ihre Gesten. Dieses Blickverhalten eröffnet ebenfalls interaktionale Räume, wie oben beschrieben. Metanarrative Räume werden gestisch am Körper der Sprecherin sowie gestisch und mittels Blicks links von ihr und vor der rechten Körperhälfte verankert. Der narrative Raum wird gestisch und mittels Blickverhaltens vor dem Oberkörper der Sprecherin und über dem Kopf etabliert. Der Reflexionsraum wird mittels Blicks vor dem Körper links oder diagonal nach oben links festgelegt. Der neutrale Raum wird nur gestisch, vor oder am Körper eröffnet. Die konstruierten Räume schließen immer die an der Etablierung beteiligten Artikulatoren mit ein.

Des Weiteren zeigt Abbildung 3, dass a) die Sprecherin nicht den gesamten, sie umgebenden realen Raum zur Etablierung konzeptueller Räume nutzt und b) die etablierten Räume simultan konstruiert werden können (,lamination“, Haviland 2000; „,blending of spaces“, Liddell 2000). Was die Visualisierung der im Beispiel etablierten Räume jedoch nicht zeigt, ist, wie sich diese Räume dynamisch und multimodal entfalten. Dieser Aspekt soll mithilfe von Abbildung 4 aufgeschlüsselt und visualisiert werden.
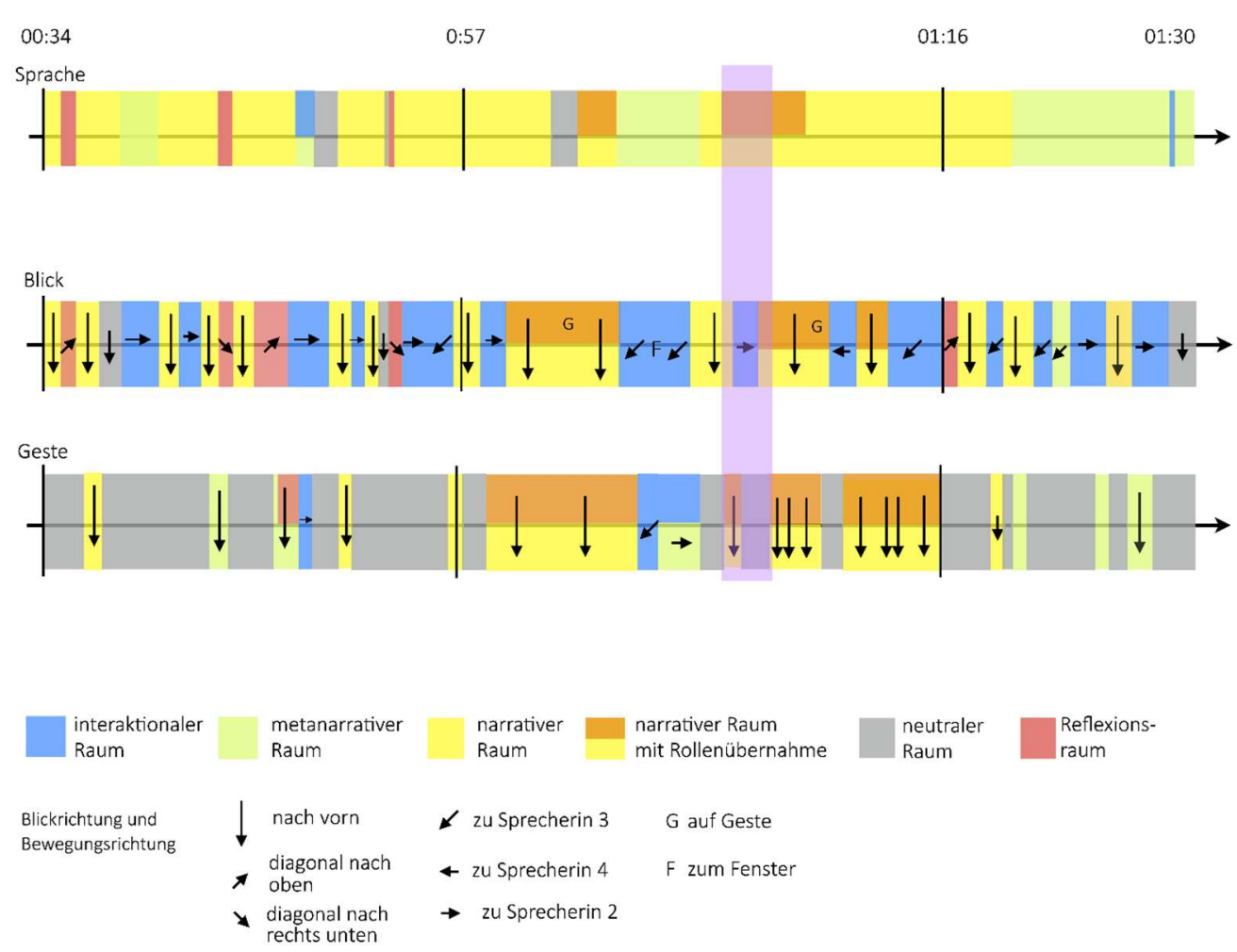

Abbildung 4: Simultane, temporale und multimodale Konstruktion von Erzählräumen

Abbildung 4 ermöglicht keine detaillierte, d. h. mikroanalytische Rekonstruktion multimodaler Bedeutungsherstellung in der untersuchten Erzählung, jedoch kann sie die Dynamik und Prozessualität der multimodalen Herstellung konzeptueller Räume sichtbar machen. Dabei zeigt sich, dass die drei untersuchten Modalitäten in ihrer temporalen Struktur unterschiedliche Räume von unterschiedlicher Dauer konstruieren. Ferner können sie simultan verschiedene 
Räume etablieren, aber auch einen konzeptuellen Raum erzeugen. Im Folgenden werden zwei Beispiele vorgestellt, die diesen Aspekt illustrieren.

Wir beginnen mit einem Beispiel, in der die Sprecherin unterschiedliche Räume konstruiert und adressieren den Abschnitt, der in Abbildung 4 violett unterlegt ist. Es zeigt sich, dass die Sprecherin verbal und gestisch einen narrativen Raum mit Rollenübernahme erzeugt und gleichzeitig mittels Blicks einen interaktionalen Raum eröffnet. Sprachlich imitiert die Sprecherin ihre Schwester, die versucht hatte, über das Fenster in ihre Wohnung zu gelangen und nun ihrer Oma, die in der Wohnung stand, antwortet Oma ich hab doch $n$ schlÜssel vergessn. Dabei reproduziert die Sprecherin Lautstärke, Dialekt und Intonation ihrer Oma. Der erste Äußerungsteil Oma ich hab doch wird begleitet von einer sehr lockeren, beidhändig ausgeführten nach oben gerichteten Hand, die die Geste ihrer Schwester nachahmt. Der Blick ist nach vorn gerichtet. Hier imitiert die Sprecherin sprachlich und körperlich ihre Schwester. Dies ändert sich während des zweiten Äußerungsteils ( $n$ schlÜssel vergessn), bei dem sprachlich immer noch die Schwester der Erzählerin imitiert wird, sich die Hände jedoch in der Ruheposition befinden und somit einen neutralen Raum etablieren. Während der Produktion dieses Äußerungsteils wandert zudem der Blick der Sprecherin in Richtung Ko-Partizipantin 2 (siehe Abbildung 4) und eröffnet damit einen interaktionalen Raum. Zusammenfassend lässt sich sagen, dass im ersten Teil der fokalen Äußerung alle untersuchten Modalitäten einen narrativer Raum mit Rollenübernahme konstruieren. Im zweiten Teil der Äußerung werden unterschiedliche Räume hergestellt, nämlich sprachlich ein narrativer Raum, gestisch ein neutraler Raum und mittels Blick ein interaktionaler Raum.

Dieses Beispiel steht exemplarisch für den Prozess der multimodalen Herstellung konzeptueller Räume, denn wie Abbildung 4 zeigt, ist ein sehr schneller Wechsel zwischen den einzelnen Perspektiven auf der Ebene körperlicher Modalitäten zu beobachten, jedoch nicht auf der sprachlichen. Das heißt, während die Sprecherin sprachlich hauptsächlich narrative Räume erzeugt, etabliert sie alternierend mithilfe ihres Blickes metanarrative- und interaktionale Räume sowie Reflexionsräume. Der neutrale Raum wird mittels Blickverhaltens nur selten erzeugt. Gestisch eröffnet die Sprecherin hauptsächlich narrative, metanarrative und neutrale Räume. Der interaktionale Raum und der Reflexionsraum werden gestisch nur selten etabliert.

Ein weiterer Aspekt, der sich über die temporale Orchestrierung der verschiedenen Modalitäten rekonstruieren lässt, ist, dass im mittleren Teil der Erzählung (00:57-01:16), die Klimax, a) sprachliche Rollenübernahmen zu beobachten sind, b) die Häufigkeit an Gesten zunimmt, die c) verstärkt Rollenübernahmen verkörpern. Diese Beobachtungen bestätigen Analysen, wonach sich die Klimax durch eine Dichte verbaler, vokaler und visueller Ressourcen auszeichnet. Diese Dichte an Modalitäten kann dabei die affektive Involviertheit von Erzähler*innen und Ko-Partizipant*innen sichtbar machen, wie Selting (2017: 29) bereits zeigte:

In order to make climaxes with affect displays recognizable as such within the local sequential context, storytellers deploy verbal, vocal, and visible devices [...] that are marked or salient in relation to their surrounding units.

(Selting 2017: 29) 
Im Folgenden werden wir den von Selting angesprochenen Aspekt der Salientsetzung explorieren und die Klimax der vorgestellten Erzählung mikroanalytisch untersuchen. Dabei wird erneut die temporale Struktur der einzelnen Modalitäten im Vordergrund stehen.

\subsection{Salientsetzen von Informationen}

Im vorhergehenden Abschnitt wurde die Temporalität von konzeptuellen Räumen sowie ihre multimodale Etablierung thematisiert. Im folgenden Abschnitt liegt der Fokus auf der engen Verzahnung der Modalitäten bei der Vermittlung semantischer Informationen, die in Abbildung 4 bereits sichtbar geworden ist. Dabei gehen wir genauer auf die Klimax der Erzählung ein, die durch eine verstärkte gestische Konstruktion des narrativen Raumes mittels Rollenübernahme seitens der Sprecherin gekennzeichnet ist. Durch die verstärkte Rollenübernahme hebt sich die Phase, die auf den Höhepunkt der Erzählung zuläuft, stark von vorhergehenden Segmenten ab und wird als solches auch für die Ko-Partizipant*innen erkennbar.

The climax of the story is conceived of (...) as a displayed point of higher emotive involvement, a 'high point' of the story, which makes relevant the recipients' affiliative responses. It thus needs to be constructed and made recognizable by the storyteller through his or her conduct towards the recipient(s). Its specific meaning and import for the story and the interaction seems to be interpretable by the recipients due to the practices and resources deployed to construct it, in conjunction with shared sociocultural knowledge. One of the tasks to be carried out in storytelling is thus for the storyteller to make recognizable the climax of the story in order to enable the recipients to infer when to respond as made relevant and thus co-construct the climax and hence the story.

(Selting 2017: 2)

In der sich temporal entfaltenden Phase, die auf die Klimax zusteuert, ist zudem eine multimodale Salienzstruktur zu erkennen, die bestimmte semantische Informationen hervorhebt und in den Fokus der Aufmerksamkeit rückt und so die Klimax vorbereitet. Auf diesen Aspekt gehen wir im Folgenden genauer ein. Die zeitliche Struktur der Modalitäten visualisieren wir dabei mit Hilfe der KeaTa-Methode (siehe Abbildung 5). Die Hervorhebung von Informationen wird mittels der MFA-Methode rekonstruiert (siehe Abbildung 7).

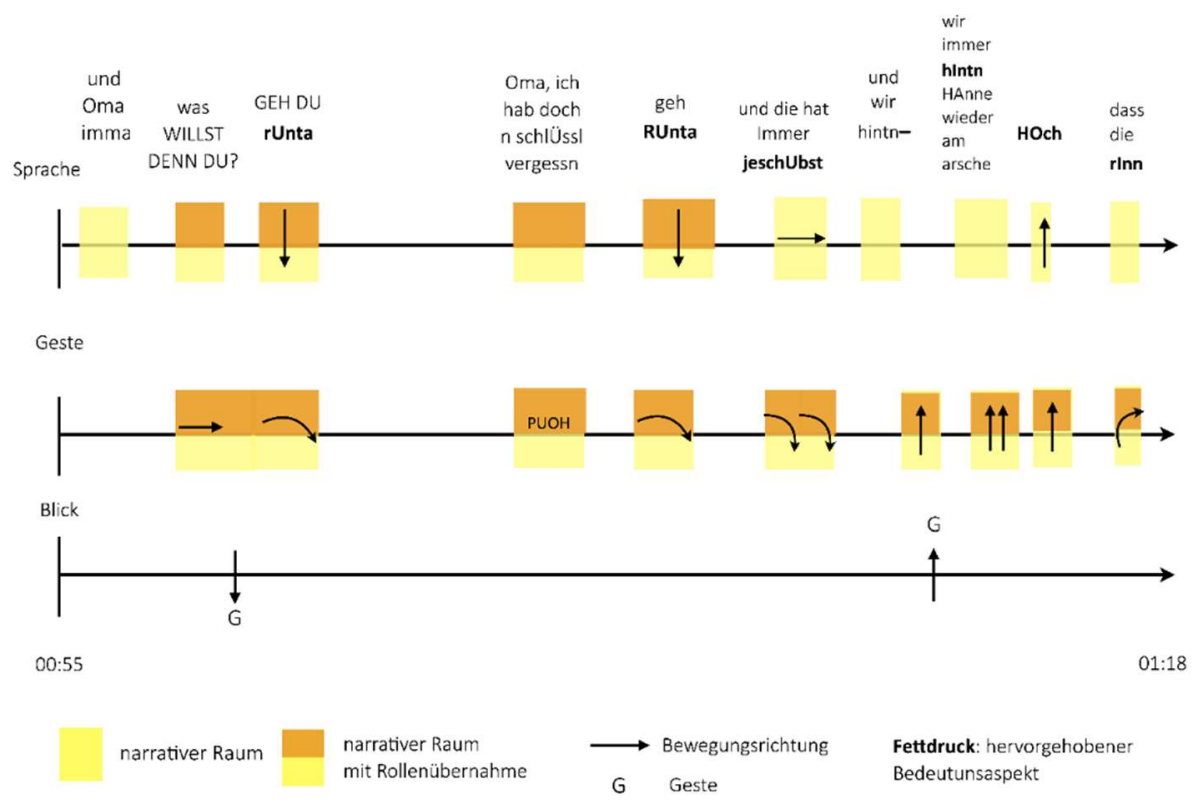

Abbildung 5: Bedeutungsentwicklung (Phase, die zur Erzählklimax führt) 
Abbildung 5 erfasst die dynamische Entwicklung von Bedeutung in der Phase der Erzählklimax, d. h. die Phase die auf die Klimax zusteuert und die Erzählklimax selbst (siehe Transkript im Anhang). Die Sprecherin beschreibt die Situationen, in der ihre Schwester versucht, über das Fenster in ihre Wohnung zu gelangen und die Oma der Sprecherin bemüht ist, sie davon abzuhalten. Gleichzeitig imitiert die Sprecherin verbal und körperlich beide Charaktere sowie sich selbst, die ebenfalls an der Situation beteiligt ist.

Die Erzählphase beginnt mit der sprachlichen Ankündigung einer Rollenübernahme (und Oma imma), woraufhin die Sprecherin ihre Oma sprachlich wie gestisch nachahmt. Wie bereits eingangs dieses Kapitels erläutert, imitiert sie ihre Äußerungen sowohl auf der Ebene sprachlicher Konstruktionen, Semantik, Dialekt und Prosodie (was willst DENN DU?). Während der Äußerung des Personalpronomens $d u$ führt sie eine beidhändige Geste aus, die die Handlung des Schubsens nachahmt (siehe Abbildung 6). Dabei sind die Hände flach, die Handinnenflächen diagonal vom Körper weg orientiert und die Bewegung wird geradlinig vom Körper weg und nach oben ausgeführt. Die Geste ist im zentralen Gestenraum positioniert und wird anschlieBend gehalten. Während des Haltens richtet die Sprecherin den Blick auf ihre Hände und setzt sie somit relevant für ihre Ko-Partizipantinnen. Denn nicht nur die Aufmerksamkeit der Sprecherin, sondern auch die ihrer Ko-Partizipantinnen ist auf die Geste gelenkt. Es operiert das Interaktive Prinzip der Hervorhebungsstrategien nach Müller/Tag (2010; siehe Kapitel 2).

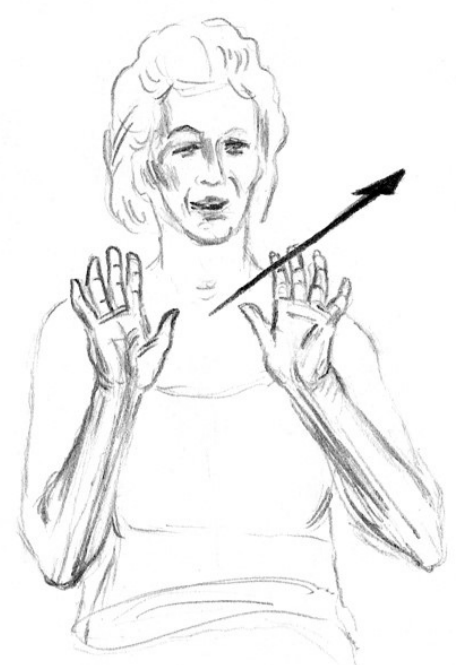

Abbildung 6: Nachgeahmte Handlung des Schubsens

Im weiteren Verlauf der Erzählung hält die Sprecherin ihre Rollenübernahme aufrecht. Sie imitiert nochmals das sprachliche und körperliche Verhalten ihrer Oma als sie sagt GEH DU rUnta und eine ähnliche Geste wie die vorhergehende ausführt (siehe Abbildung 6). Diese weist die gleichen Formparameter wie die vorherige Geste auf, zeigt jedoch eine Variation in der Bewegungsrichtung, denn sie wird nicht geradlinig nach vorn, sondern in einer leicht gebogenen Bewegung nach unten ausgeführt. Diese Bewegungsrichtung wird sprachlich ausgedrückt und simultan gestisch verkörpert (siehe Abbildung 5). Aufgrund dieser multimodalen Konstruktion von Bedeutung argumentieren wir, dass in diesem Moment des Sprechens und Gestikulierens die Bewegungsrichtung und nicht das Bewegungsmuster salient ist. Demnach operiert hier das Ikonizitätsprinzip (ibd.), wonach Bedeutung, die in mehreren Modalitäten ausgedrückt wird, in 
den Fokus der Aufmerksamkeit rückt. Die neben der Sprecherin sitzende Ko-Partizipantin ratifiziert die multimodale Äußerung mit einem Lachen. Die Sprecherin fährt fort und vermittelt einige metanarrative Informationen bezüglich des Fenster- und Wohnungstyps. Im Anschluss daran rekonstruiert sie den narrativen Raum, indem sie eine weitere Rollenübernahme ankündigt (und hanne immer), die sie anschließend sprachlich und körperlich mit der Äußerung Oma $i c h$ hab doch $n$ schlÜssel vergessn vollzieht. Diese Äußerung wird begleitet von einer sehr lockeren nach oben gerichteten Hand, die dann in die Ruheposition geführt wird. Ohne weitere Ankündigung wechselt die Sprecherin anschließend in die Rolle ihrer Oma, wie anhand sprachlicher und gestischer Parameter zu beobachten ist. Mit der kurzen sprachlichen Äußerung geh RUnta, die von einer weiteren manuellen Schubsbewegung begleitet wird, fokussiert sie nochmals die Handlung des Nach-Unten-Schubsens, wobei abermals die Bewegungsrichtung hervorgehoben wird. So akzentuiert sie das Adverb runter und führt simultan eine gestische Bewegung nach unten aus (Ikonizitätsprinzip). Die nach unten gerichtete Bewegung wird folglich hervorgehoben und salient gesetzt. Auch hier dokumentiert die neben der Sprecherin sitzende Interaktionspartnerin ihr Verstehen mittels eines lauten Lachens. Im weiteren Verlauf kulminiert die Erzählung in der Verkörperung mehrerer manueller Schubs- und Drückbewegungen. Zunächst ahmt die Sprecherin zwei Schubsbewegungen nach, die simultan zur verbalen Äußerung und die hat Immer jeschubst ausgeführt werden und dieselbe Form wie die vorhergehenden Gesten zeigen. Mit der sprachlichen Referenz auf die Handlung des Schubsens wird erstmals das Handlungsmuster und nicht allein die Bewegungsrichtung salient gesetzt. Dieses wurde zwar in den vorhergehenden Gesten verkörpert, aber nicht durch die gleichzeitige sprachliche Referenz hervorgehoben (Ikonizitätsprinzip). ${ }^{6}$ Der Fokus der Aufmerksamkeit wechselt demgemäß von der Bewegungsrichtung zum Handlungsmuster. Dieser Fokus wird jedoch im weiteren Verlauf der Erzählung nicht aufrechterhalten, denn die Sprecherin richtet ihre Aufmerksamkeit nun erneut auf die Bewegungsrichtung der imitierten Handlungen. Sie beginnt eine weitere verbale Äußerung (und wir hintn), unterbricht diese und produziert eine Geste, die eine nach oben gerichtete Schubsbewegung darstellt. Diese Geste füllt die durch den Abbruch entstandene syntaktische und semantische Lücke (für eine genaue Analyse siehe Ladewig 2014a; 2019; 2020). Hier wird nun die Geste samt der Bewegungsrichtung in den Vordergrund gerückt, da sie sprachliche Informationen substituiert (Semantisches und Syntaktisches Prinzip nach Müller/Tag 2010) und die Ko-Partizipantinnen damit aufgefordert sind, relevante Informationen zur Verständnissicherung in einer anderen Modalität, hier der gestischen, zu suchen. Des Weiteren richtet die Sprecherin ihren Blick auf die Geste und hebt sie damit hervor (Interaktives Prinzip, ibd.). Ferner wird die Geste in Opposition zu der vorab hervorgehobenen Bewegungsrichtung nach unten gesetzt, da nun erstmals eine nach oben gerichtete Bewegung ausgeführt wird (siehe Abbildung 5). Mit der Konstruktion dieses Gegensatzes wird die nach oben ausgeführte Bewegung hervorgehoben und salient gesetzt. Obwohl dieser Aspekt nicht Teil der Differenzierung von Müller/Tag (2010) ist, ordnen wir dieses Phänomen dem Ikonizitätsprinzip zu. Zu ergänzen ist, dass die rein gestisch übermittelten Informationen

\footnotetext{
${ }^{6}$ Was sich hier ebenso sehr gut beobachten lässt, ist die Konstruktion der narrativen Räume auf sprachlicher und gestischer Ebene. Auf sprachlicher Ebene wird die Rollenübernahme nicht aufrechterhalten, jedoch auf der gestischen.
} 
von einer der Ko-Partizipantinnen verstanden wurde, was anhand der sich anschließenden Ratifizierung in Form von herzlichem Lachen sowie der Nachfrage Echt rekonstruierbar ist. ${ }^{7}$

Im Anschluss an die Erzählklimax elaboriert die Sprecherin die Handlung des Nach-Oben-Drückens (wir immer hIntn HAnne wieder am Arsche wir immer hinten immer Hanne am arsche hHOch dass die rInn) und hebt sprachlich wie gestisch die Bewegung nach oben hervor (Ikonizitätsprinzip).

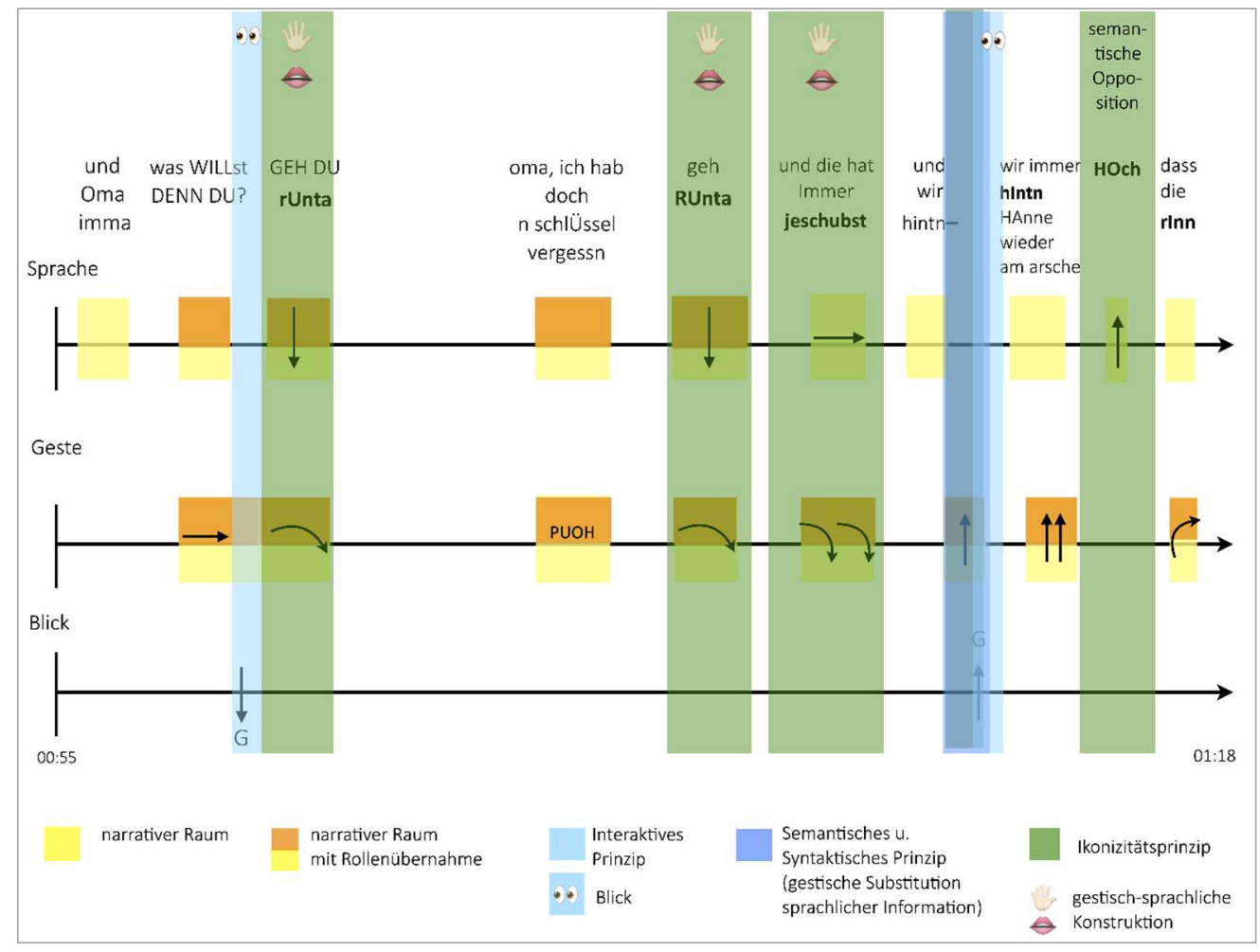

Abbildung 7: Visualisierung der multimodalen Salienzstruktur

Abbildung 7 fasst die eben beschriebene temporale Entwicklung der multimodalen Salienzstruktur zusammen. Die operierenden Prinzipien, die diese Salienzstruktur hervorbringen, werden durch farbliche Blöcke und Symbole veranschaulicht. Es zeigt sich, dass das Ikonzitätsprinzip (grüne Markierung in Abbildung 7) ab der ersten multimodalen Äußerung (was WILLst $D E N N D U$ + beidhändige Geste) operiert und, wie eben beschrieben, im Verlauf der Erzählung einzelne semantische Facetten sprachlich-gestischer Bedeutung hervorhebt. Genauer gesagt, wird in der temporalen Entfaltung multimodaler Bedeutung die Bewegungsrichtung nach unten mehrfach salient und am Höhepunkt der Erzählung in Opposition zu einer gestischen Bewegung nach oben gesetzt. Das Semantische und Syntaktische Prinzip (dunkelblaue Markierung in Abbildung 7) operiert ebenfalls am Höhepunkt der Erzählung. Hier füllt die Geste eine semantische und syntaktische Lücke, was bedeutet, dass die für das Herstellen von Intersubjektivität notwendigen Informationen rein gestisch übermittelt werden. Durch die semantische und syntaktische Integration der Geste in die verbale Äußerung und wir hintn wird gestische Bedeutung hervorgehoben und in den Fokus der Aufmerksamkeit gerückt. Das Interaktive Prinzip

\footnotetext{
${ }^{7}$ Wie Perzeptionsexperimente zudem zeigten, wird sowohl das Handlungsmuster als auch die Bewegungsrichtung als relevante Information perzipiert (Ladewig 2019, 2020). Diese Befunde unterstützen die hier vorgestellte Analyse der dynamischen Bedeutungsentfaltung.
} 
(hellblaue Markierung in Abbildung 7), das anhand der Blickrichtung empirisch rekonstruierbar ist, operiert an zwei Stellen der Erzählung. Interessanterweise ist es aktiv, kurz bevor die Sprecherin das erste Mal die Bewegungsrichtung nach unten sprachlich und gestisch salient setzt (GEH DU rUnta + beidhändige Geste). Hier ist der Blick nach unten auf die Geste gerichtet, was durch den nach unten gerichteten Pfeil in Abbildung 7 symbolisiert wird. Außerdem ist es aktiv, wenn die Sprecherin die zweite Bewegungsrichtung, die Bewegung nach oben, etabliert (siehe nach oben gerichteter Pfeil in Abbildung 7).

Mit dieser sich temporal entfaltenden Salienzstruktur geht auch eine sich temporal entfaltende Aufmerksamkeitssteuerung auf bestimmte semantische Aspekte der Erzählung einher. Die Sprecherin hebt sprachlich und körperlich semantische Aspekte hervor und rückt sie dabei nicht nur für sich, sondern auch für ihre Ko-Partizipantinnen in den Fokus der Aufmerksamkeit. Der daraus resultierende Fokus auf zwei Bewegungsrichtungen trägt wesentlich zur Erzählklimax und zur Pointe der Erzählung bei. Dass diese verstanden wurde, zeigt sich in der Ratifizierung durch eine Ko-Partizipantin, die sich in herzhaftem Lachen ausdrückt (siehe Transkript im Anhang).

\section{$4 \quad$ Konklusion}

Die in diesem Artikel vorgestellte Studie untersuchte anhand einer Erzählung die temporale Entfaltung und multimodale Orchestrierung von konzeptuellen Räumen. Dabei bewegte sich der Analysefokus von der Makroebene der gesamten Erzählung und der darauf untersuchten temporalen und multimodalen Orchestrierung konzeptueller Räume zur Mikroebene und damit dem Zusammenspiel einzelner Modalitäten in der Konstruktion von Bedeutung (am Beispiel der Erzählklimax).

Basierend auf der makroanalytischen Untersuchung von Sprache, Blickverhalten und Gesten konnten fünf konzeptuelle Räume bestimmt werden - der narrative Raum (mit und ohne Rollenübernahmen), der metanarrative Raum, der interaktionale Raum, der Reflexionsraum und der neutrale Raum. Diese werden mittels unterschiedlicher Modalitäten kreiert, wobei einzelne Modalitäten aufgrund ihrer medialen Eigenschaften für die Herstellung bestimmter konzeptueller Räume stärker genutzt werden als andere. So wird der interaktionale Raum häufig mittels Blickverhaltens kreiert, wobei der narrative Raum ohne Rollenübernahme häufig sprachlich konstruiert wird. Zudem zeigte die Studie, dass konzeptuelle Räume häufig zeitgleich erzeugt werden, was aufgrund der medialen Beschaffenheit der daran beteiligten Modalitäten jedoch nicht zu Verständigungsproblemen führt (cf. Schmitts (2005) Diskussion des overlapKonzepts).

The particular temporal constraints and affordances each modal resource implies are consequential for how different resources are coordinated to produce actions [...] To produce synchronized multimodal action, participants make use of the temporal logics of the different resources, taking into account the requirements each modality places on attention and perceptibility both intra- and inter-personally.

(Deppermann/Günthner 2015: 10).

Die mikroanalytische Analyse behandelte das simultane und sequenzielle Zusammenspiel der untersuchten Modalitäten. Dabei zeigte sich, dass in der Phase der Erzählklimax, in der die multimodale Herstellung des narrativen Raumes mit Rollenübernahme dominierte, semantische 
Informationen über die multimodale und sequenzielle Erzeugung salient gesetzt wurden. Das heißt, wird Bedeutung beispielsweise sprachlich-gestisch konstruiert, wandert der Blick der Erzählerin auf ihre Hände oder füllen ihre Gesten eine semantische und syntaktische Lücke, so werden semantische Informationen hervorgehoben und für die Ko-Partizipant*innen relevant gesetzt. Bedeutung wird ,spontaneously elaborated, foregrounded, [and] made prominent for an attending co-participant" (Müller/Tag 2010: 89, Hervorhebung im Original). Intersubjektivität entsteht über die gemeinsame Konstruktion dieser sich temporal entfaltenden Salienzstruktur, die einhergeht mit einer sich temporal entfaltenden Aufmerksamkeitssteuerung.

Zusammenfassend lässt sich festhalten, dass die Konstruktion konzeptueller Räume in multimodalen Erzählungen äußerst komplex ist. Dabei übernehmen die verschiedenen Modalitäten in ihrer zeitlichen Entfaltung und basierend auf ihrer spezifischen Medialität unterschiedliche Funktionen. Jede Modalität weist eine eigene zeitliche Struktur auf (cf. u. a. Keevallik 2015), ist aber im Gebrauch mit weiteren Ausdrucksmodalitäten eng verzahnt. Sie orchestrieren gemeinsam Bedeutung im zeitlichen Verlauf der Erzählung, heben dabei einzelne Bedeutungsaspekte hervor und machen sie relevant für Ko-Partizipant*innen.

Im Hinblick auf eine methodische wie theoretische Reflexion bedeuten diese Beobachtungen, dass mit dem Fokus auf eine rein sprachliche Analyse, ein sehr großer Teil der interaktiven Bedeutungskonstitution und -aushandlung verlorenginge. Bedeutungsherstellung vollzieht sich demnach nicht ausschließlich über gesprochene Sprache, sondern stellt im Zusammenspiel der einzelnen Modalitäten sowie in deren temporalen Verlauf Relevanz für das Erzählverständnis her.

\section{Literaturverzeichnis}

Beattie, Geoffrey/Shovelton, Heather (2001): „How gesture viewpoint influences what information decoders receive from iconic gestures". In: Cave, Christian/Guaitella, Isabella/Santi, Serge (eds.): Oralite et Gestualite: Interactions et comportements multimodaux dans la communication. L'Harmattan, Paris: 283-287.

Beattie, Geoffrey/Shovelton, Heather (2002): „An experimental investigation of some properties of individual iconic gestures that mediate their communicative power". British Journal of Psychology 93/2: 179-192.

Bressem, Jana (2013): ,A linguistic perspective on the notation of form features in gestures. In: Müller, Cornelia et al. (eds.): Body - Language - Communication: An International Handbook on Multimodality in Human Interaction. Berlin/Boston, de Gruyter: 1079-1098. (= Handbooks of Linguistics and Communication Science 38.1).

Bressem, Jana/Ladewig, Silva H./Müller, Cornelia (2013): „A linguistic annotation system for gestures (LASG)". In: Müller, Cornelia et al. (eds.): Body - Language - Communication: An International Handbook on Multimodality in Human Interaction. Berlin/Boston, de Gruyter: 1098-1125. (= Handbooks of Linguistics and Communication Science 38.1).

Bressem, Jana/Ladewig, Silva H./Müller, Cornelia (2018): „Ways of expressing action in multimodal narrations - The semiotic complexity of character viewpoint depictions". In: Hübl, Anika/Steinbach, Markus (eds.): Linguistic Foundations of Narration in Spoken and Sign Languages. Amsterdam, Benjamins: 223-249. 
Brown, Amanda (2008): „Gesture viewpoint in Japanese and English: Cross-linguistic interactions between two languages in one speaker". Gesture 8/2: 256-276.

Bühler, Karl (1934): Sprachtheorie. Die Darstellungsfunktion der Sprache. Stuttgart/New York: Fischer.

Cameron, Lynne (2002): Metaphor in educational discourse. New York: Continuum.

Casey, Shannon/Emmorey, Karen (2009). „Co-speech gesture in bimodal bilinguals”. Language and Cognitive Processes 24/2: 290-312.

Cassell, Justine/McNeill, David (1991): „Gesture and the poetics of prose”. Poetics Today 12/3: 375-404.

Chafe, Wallace L. (1994): Discourse, consciousness, and time : the flow and displacement of conscious experience in speaking and writing. Chicago: University of Chicago Press.

Cienki, Alan/Müller, Cornelia (2014): Ways of viewing metaphor in gesture. In: Müller, Cornelia et al. (eds.): Body - Language - Communication: An International Handbook on Multimodality in Human Interaction. Berlin/Boston, de Gruyter: 1766-1781. (= Handbooks of Linguistics and Communication Science 38.2).

Cormier, Kearsy et al. (2012): „Lexicalisation and de-lexicalisation processes in sign languages: Comparing depicting constructions and viewpoint gestures". Language \& communication 32/4: 329-348.

Croft, William/Cruse, David Alan (2004): Cognitive Linguistics. Cambridge: Cambridge Univeristy Press.

Dancygier, Barbara (2011): The language of stories: A cognitive approach. Cambridge: Cambridge University Press.

Deppermann, Arnulf (1999): Gespräche analysieren. Eine Einführung in konversationsanalytische Methoden. Opladen: Leske und Buderich.

Deppermann, Arnulf/Günthner, Susanne (2015): ,Introduction: Temporality in Interaction”. In: Arnulf Deppermann/Günthner, Susanne (eds): Temporality in Interaction. Amsterdam, Benjamins: 1-23.

Dudis, Paul G. (2004): „Body partitioning and real-space blends”. Cognitive Linguistics 15/2: 223-238.

Duncan, Susan D. (2005): „Gesture in signing: A case study from Taiwan Sign Language”. Language and Linguistics 6/2: 279-318.

Earis, Helen/Cormier, Kearsy (2013): „Point of view in British Sign Language and spoken English narrative discourse: the example of "The Tortoise and the Hare"'. Language and Cognition 5/4: 313-343.

Ehmer, Oliver (2011): Imagination und Animation. Die Herstellung mentaler Räume durch animierte Rede. Berlin/New York: de Gruyter.

Fauconnier, Gilles (1985): Mental spaces : aspects of meaning construction in natural language. Cambridge: MIT Press.

Fauconnier, Gilles/Sweetser, Eve (1996): Spaces, Worlds, and Grammar. Chicago: University Of Chicago Press.

Fauconnier, Gilles/Turner, Mark (2002): The way we think : conceptual blending and the mind's hidden complexities. New York: Basic Books.

Fogarty-Bourget, Chloë G./Artemeva, Natasha/Fox, Janna D. (2019): „Gestural silence: An engagement device in the multimodal genre of the chalk talk lecture". In: Sancho Guinda, 
Carmen (ed.): Engagement in Professional Genres: Deference and disclosure. Amsterdam, Benjamins: 277-295.

Frederiksen, Anne Therese (2017): „Separating viewpoint from mode of representation in iconic co-speech gestures: insights from Danish narratives". Language and Cognition 9/4: $677-708$.

Fricke, Ellen (2012): Grammatik multimodal. Wie Gesten und Wörter zusammenwirken. Berlin: de Gruyter.

Fuchs, Thomas (2017): „Intercorporeality and interaffectivity”. In: Meyer, Christian/Streeck, Jürgen/Jordan, Scott (Eds.): Intercorporeality: Emerging Socialities in Interaction. New York, Oxford University Press: 194-209.

Garfinkel, Harold (1967): Studies in ethnomethodology. Englewood Cliffs: Prentice-Hall.

Gibbs, Raymond W. (2018): „Our Metaphorical Experiences of Film”. In: Greifenstein, Sarah et al. (eds.): Cinematic Metaphor II. Reflections on a Transdisciplinary Framework. Berlin/Boston, de Gruyter: 120-140

Goffman, Erving (1981): Forms of talk. Philadelphia: University of Pennsylvania Press.

Goodwin, Charles (1981): Conversational Organization: Interaction Between Speakers and Hearers. New York: Academic Press.

Gülich, Elisabeth (2005): „Krankheitserzählungen“. In: Neises, Mechthild (ed.): Psychosomatische Gesprächsführung in der Frauenheilkunde: ein interdisziplinärer Ansatz zur verbalen Intervention. Stuttgart, Wissenschaftliche Verlagsgesellschaft: 73-89.

Gülich, Elisabeth (2007): „Mündliches Erzählen: narrative und szenische Rekonstruktion“. In: Lubs, Sylke (ed.): Behutsames Lesen. alttestamentliche Exegese im interdisziplinären Methodendiskurs. Christof Hardmeier zum 65. Geburtstag. Leipzig: Evangelische Verlagsanstalt: $35-62$.

Gülich, Elisabeth (2008): „Alltagliches erzahlen und alltagliches Erzahlen“. Zeitschrift für Germanistische Linguistik 36/3: 403-426.

Gülich, Elisabeth/Hausendorf, Heiko (2000): Vertextungsmuster: Narration. In: Brinker, Klaus (ed.): Text-und Gesprächslinguistik. 1. Halbbd. Berlin, de Gruyter: 369-385. (= Handbücher zur Sprach- und Kommunikationswissenschaft 16)

Gullberg, Marianne/Kita, Sotaro (2009): „Attention to speech-accompanying gestures: Eye movements and information uptake". Journal of nonverbal behavior 33/4: 251-277.

Gunter, Thomas C./Weinbrenner, J. E. Douglas/Holle, Henning (2015): „Inconsistent use of gesture space during abstract pointing impairs language comprehension". Frontiers in Psychology 6/80. frontiersin.org/articles/10.3389/fpsyg.2015.00080/full [23.10.2020].

Haddington, Pentti et al. (eds.) (2014): Multiactivity in social interaction: Beyond multitasking. Amsterdam: Benjamins.

Hausendorf, Heiko/Quasthoff, Uta M. (1996): Sprachentwicklung und Interaktion: eine linguistische Studie zum Erwerb von Diskursfähigkeiten. Wiesbaden: Springer.

Haviland, John B. (2000): „Pointing, gesture spaces and mental maps”. In: McNeill, David (ed.): Language and Gesture”. Cambridge, Cambridge University Press: 13-46.

Heller, Vivien (2018): „Jenseits des Hier und Jetzt. Multimodale Praktiken der Versetzung in Erzählinteraktionen kleiner Kinder“. In: König, Katharina/Oloff, Florence (eds.): Erzählen multimodal. Gesprächsforschung - Online-Zeitschrift zur verbalen Interaktion 19: 242-274. 
gespraechsforschung-online.de/fileadmin/dateien/heft2018/erzaehlen-multimodal.pdf [23.10.2020].

Horst, Dorothea (2018): Meaning-Making and Campaign Advertising. The Dynamics of Audiovisual Figurativity in German and Polish Campaign Commercials. Berlin/Boston: de Gruyter.

Horst, Dorothea et al. (2014): „Gestures as interactive expressive movement: Inter-affectivity in face-to-face communication”. In: Müller, Cornelia et al. (eds.): Body - Language Communication: An International Handbook on Multimodality in Human Interaction,. Berlin/Boston, de Gruyter: 2112-2125. (= Handbooks of Linguistics and Communication Science 38.2).

Jakobson, Roman (1966): Selected Writings. Slavic Epic Studies. The Hague: Mouton.

Jensen, Thomas Wiben (2017): „Doing Metaphor: An Ecological Perspective on Metaphoricity in Discourse". In: Hampe, Beate (ed.): Metaphor: Embodied Cognition and Discourse. Cambridge, Cambridge University Press: 257-276.

Kallmeyer, Werner (1981): „Aushandlung und Bedeutungskonstitution“. In: Schröder, Peter/Steger, Hugo (eds.): Dialogforschung. Jahrbuch 1980 des Instituts für deutsche Sprache. Düsseldorf, Schwann: 89-127.

Kappelhoff, Hermann/Müller, Cornelia (2011): „Embodied meaning construction. Multimodal metaphor and expressive movement in speech, gesture, and feature film". Metaphor in the Social World 1/2: 121-153.

Keevallik, Leelo (2015): "Coordinating the temporalities of talk and dance". In: Arnulf Deppermann/Günthner, Susanne (eds): Temporality in Interaction. Amsterdam, Benjamins: 309-336.

Kendon, Adam (1972): „Some relationships between body motion and speech: An analysis of an example". In: Siegman, Aron Wolfe/Pope, Benjamin (eds.): Studies in Dyadic Communication. New York, Elsevier: 177-210.

Kendon, Adam (1995): „Gestures as illocutionary and discourse structure markers in Southern Italian conversation". Journal of Pragmatics 23: 247-279.

Kolter, Astrid, et al. (2012): „Body memory and emergence of metaphor in movement and speech. An interdisciplinary case study". In: Koch, Sabine et al. (eds): Body Memory, Metaphor, and Movement. Amsterdam/Philadelphia, Benjamins: 201-226.

König, Katharina/Oloff, Florence (2018): Erzählen multimodal. Gesprächsforschung - OnlineZeitschrift zur verbalen Interaktion 19: 207-241. gespraechsforschung-online.de/filead$\mathrm{min} /$ dateien/heft2018/erzaehlen-multimodal.pdf [23.10.2020].

Labov, William (1972): „The transformation of experience in narrative syntax”. In: Labov, William (ed.): Language in the inner city. Studies in the Black vernacular. Philadelphia, Philadelphia University Press: 354-396.

Ladewig, Silva H. (2014a): „Creating multimodal utterances: The linear integration of gestures into speech". In: Müller, Cornelia et al. (eds.): Body - Language - Communication. An International Handbook on Multimodality in Human Interaction. Berlin/Boston, de Gruyter: 1662-1677. (= Handbooks of Linguistics and Communication Science 38.2).

Ladewig, Silva H. (2014b): „Recurrent gestures. ” In: Müller, Cornelia et al. (eds.): Body Language - Communication. An International Handbook on Multimodality in Human 
Interaction. Berlin/Boston, de Gruyter: 1558-1575. (= Handbooks of Linguistics and Communication Science 38.2).

Ladewig, Silva H. (2019): „Konzeptuelle Integration von Sprache und Geste am Beispiel gestisch vervollständigter Äußerungen“. Mitteilungen des Deutschen Germanistenverbandes 66/4: 393-401.

Ladewig, Silva H. (2020): Integrating Gestures. The Dimension of Multimodality in Cognitive Grammar. Berlin/Amsterdam: de Gruyter.

Liddell, Scott K. (2000): „Blended spaces and deixis in sign language”. Language and gesture 2: 331 .

Lucius-Hoene, Gabriele/Deppermann, Arnulf (2002): Narrative Identität und Positionierung. Ein Arbeitsbuch zur Analyse narrativer Interviews. Wiesbaden: Springer Fachmedien.

Maury-Rouan, Claire (2011): „Investigating nonverbal parameters of the participation framework". In: Stam, Gale/Ishino, Mika (eds.): Integrating gestures: The interdisciplinary nature of gesture. Amsterdam/Philadelphia, Benjamins: 309-320.

Mayerthaler, Willi (1980): „Ikonismus in der Morphologie“. Zeitschrift für Semiotik 2: 19-37.

McClave, Evelyn Z. (2000): „Linguistic functions of head movements in the context of speech”. Journal of Pragmatics 32: 855-878.

McNeill, David (1992): Hand and mind. What gestures reveal about thought. Chicago: University of Chicago Press.

Merleau-Ponty, Maurice (1962/2005): Phenomenology of perception. London/New York: Routledge.

Müller, Cornelia (1998): Redebegleitende Gesten: Kulturgeschichte, Theorie, Sprachvergleich. Berlin: Arno Spitz.

Müller, Cornelia (2003): „,On the gestural creation of narrative structure: A case study of a story told in conversation". In: Rector, Monica/Poggi, Isabella/Trigo, Nadine (eds.): Gestures: Meaning and Use. Porto, Universidade Fernando Pessoa: 259-265.

Müller, Cornelia (2004): „Forms and uses of the Palm Up Open Hand. A case of a gesture family?’. In: Müller, Cornelia/Posner, Roland (eds.): Semantics and Pragmatics of everyday gestures. Berlin, Weidler: 234-256.

Müller, Cornelia (2007): „A dynamic view on gesture, language and thought”. In: Duncan, Susan D./Cassell, Justine/Levy, Elena T. (eds.): Gesture and the dynamic dimension of language. Amsterdam/Philadelphia, Benjamins: 109-116.

Müller, Cornelia (2008a): Metaphors dead and alive, sleeping and waking: a dynamic view. Chicago: University of Chicago Press.

Müller, Cornelia (2008b): „What gestures reveal about the nature of metaphor”. In: Cienki, Alan/Müller, Cornelia (eds.): Metaphor and Gesture. Amsterdam, Benjamins: 249-275.

Müller, Cornelia (2013): „Gestures as a medium of expression: The linguistic potential of gestures". In: Müller, Cornelia et al. (eds.): Body - Language - Communication. An International Handbook on Multimodality in Human Interaction. Berlin/Boston, de Gruyter: 202-217. (= Handbooks of Linguistics and Communication Science 38.2).

Müller, Cornelia (2014): „Gestural modes of representation as techniques of depiction”. In: Müller, Cornelia et al. (eds.): Body - Language - Communication. An International Handbook on Multimodality in Human Interaction. Berlin/Boston, de Gruyter: 1687-1702. (= Handbooks of Linguistics and Communication Science 38.2). 
Müller, Cornelia (2017): „Waking Metaphors. Embodied Cognition in Multimodal Discourse”. In: Hampe, Beate (ed.): Metaphor. Embodied Cognition in Discourse. Cambridge, Cambridge University Press: 291-316.

Müller, Cornelia/Bressem, Jana/Ladewig, Silva H. (erscheint): Gesture and Language (Textbook). London/New York: Routledge.

Müller, Cornelia/Kappelhoff, Hermann (2018): Cinematic metaphor: experience-affectivitytemporality. Berlin/Boston: de Gruyter.

Müller, Cornelia/Ladewig, Silva H. (2013): „Metaphors for sensorimotor experiences. Gestures as embodied and dynamic conceptualizations of balance in dance lessons". In: Borkent, Mike/Dancygier, Barbara/Hinnell, Jennifer (eds.): Language and the Creative Mind. Stanford, CSLI: 295-324.

Müller, Cornelia/Schmitt, Christina (2015): „Audio-visual metaphors of the financial crisis: meaning making and the flow of experience". Revista Brasileira de Linguística Aplicada 15/2: 311-342.

Müller, Cornelia/Tag, Susanne (2010): „The Dynamics of Metaphor. Foregrounding and Activating Metaphoricity in Conversational Interaction”. Cognitive Semiotics 6: 85-120.

Parrill, Fey (2009): „Dual viewpoint gestures”. Gesture 9/3: 271-289.

Parrill, Fey (2010): „Viewpoint in speech-gesture integration: Linguistic structure, discourse structure, and event structure". Language and Cognitive Processes 25/5: 650-668.

Parrill, Fey (2012): „Interactions between discourse status and viewpoint in co-speech gesture”. In: Dancygier, Barbara/Sweetser, Eve (eds.): Viewpoint in language: A multimodal perspective. Cambridge, Cambridge Univeristy Press: 97-112.

Payrató, LLuís/Teßendorf, Sedinha (2014): „Pragmatic gestures”. In: Müller, Cornelia et al. (eds.): Body - Language - Communication: An International Handbook on Multimodality in Human Interaction. Berlin/Boston, de Gruyter: 1531-1539. (= Handbooks of Linguistics and Communication Science 38.2).

Pfau, Roland/Steinbach, Markus (2006): „Modality-independent and Modality-specific Aspects of Grammaticalization in Sign Language". Linguistics in Potsdam 24/3: 3-98.

Pörings, Ralf/Schmitz, Ulrich (2003): Sprache und Sprachwissenschaft: eine kognitiv orientierte Einführung. Tübingen: Narr.

Quasthoff, Uta M. (1980): Erzählen in Gesprächen: Linguistische Untersuchungen zu Strukturen und Funktionen am Beispiel einer Kommunikationsform des Alltags. Tübingen: Narr.

Quasthoff, Uta M. (2001): „Erzählen als interaktive Gesprächsstruktur“. In: Brinker, Klaus et al. (eds.): Text-und Gesprächslinguistik. Ein internationales Handbuch zeitgenössischer Forschung. Berlin/New York, de Gruyter: 1293-1309. (= Handbücher zur Sprach- und Kommunikationswissenschaft 16/2)

Quinto-Pozos, D./Parrill, F. (2015): „Signers and co-speech gesturers adopt similar strategies for portraying viewpoint in narratives". Top Cogn Sci 7/1: 12-35.

Sacks, Harvey (1992b): „Long Sequences”. In: Jefferson, Gail (ed.): Lectures on conversation. Cambridge, Blackwell: 354-360.

Sacks, Harvey/Schegloff, Emanuel A./Jefferson, Gail (1974): „A Simplest Systematics for the Organization of Turn-Taking for Conversation”. Language 50/4: 696-735.

Schegloff, Emanuel A. (1998): „Body torque”. Social Research 65/3: 535-596. 
Schegloff, Emanuel A. (2000): „On granularity”. Annual Review of Sociology 26/1: 715-720. Schmitt, Christina/Greifenstein, Sarah/Kappelhoff, Hermann (2014): „Expressive movement and metaphoric meaning making in audio-visual media”. In: Müller, Cornelia et al. (eds.): Body - Language - Communication. An International Handbook on Multimodality in Human Interaction. Berlin/Boston, de Gruyter: 2092-2112. (= Handbooks of Linguistics and Communication Science 38.2).

Schmitt, Reinhold (2005): „Zur multimodalen Struktur von turn-taking“. Gesprächsforschung - Online-Zeitschrift zur verbalen Interaktion Ausgabe 6: 17-61. gespraechsforschungozs.de/heft2005/ga-schmitt.pdf [24.10.2020].

Schütze, Fritz (1994): „Das Paradoxe in Felix“ Leben als Ausdruck eines ,Wilden“ Wandlungsprozesses“. In: Koller, Hans-Christoph/Kokemohr, Rainer (eds.): Lebensgeschichte als Text: Zur biographischen Artikulation problematischer Bildungsprozesse. Weinheim, Dt. Studien: 13-60.

Selting, Margret (2017): „The display and management of affectivity in climaxes of amusing stories”. Journal of Pragmatics 111: 1-32.

Stec, Kashmiri (2012): „Meaningful shifts: A review of viewpoint markers in co-speech gesture and sign language". Gesture 12/3: 327-360.

Streeck, Jürgen (1988): „The significance of Gesture: How it is established”. IPrA Papers in Pragmatics 2/1/2: 60-83.

Streeck, Jürgen (2009): Gesturecraft: Manufacturing understanding. Amsterdam/Philadelphia: Benjamins.

Sweetser, Eve (2013): „Creativity across modalities in viewpoint construction”. In: Borkent, Mike/Dancygier, Barbara/Hinnell, Jennifer (eds.): Language and the Creative Mind. Stanford, CSLI: 239-254.

Sweetser, Eve/Sizemore, Marisa (2008): „Personal and interpersonal gesture spaces: Functional contrasts in language and gesture”. In: Tyler, Andrea/Kim, Yiyoung/Takada, Mari (eds.): Language in the Context of Use: Cognitive and Discourse Approaches to Language and Language Learning. Berlin, de Gruyter: 25-52.

Sweetser, Eve/Stec, Kashmiri (2016): „Maintaining multiple viewpoints with gaze”. In: Dancygier, Barbara/Lu , Wei-lun/Verhagen, Arie (eds.): Viewpoint and the fabric of meaning: Form and use of viewpoint tools across languages and modalities. Berlin/Boston, de Gruyter: 237-257.

Thompson, Sandra A./Suzuki, Ryoko (2014): „Reenactments in conversation: Gaze and recipiency". Discourse Studies 16/6: 816-846.

Tuite, Kevin (1993 ): „The production of gesture”. Semiotica 93/1-2: 83-105.

Wilcox, Sherman (2004). „Gesture and language”. Gesture 4/1: 43-73.

Wittenburg, Peter et al. (2006): ELAN: a professional framework for multimodality research. Paper presented at the Proceedings of 5th International Conference on Language Resources and Evaluation (LREC 2006). 


\section{Anhang A: Transkript der Erzählung}

\begin{tabular}{|c|c|c|}
\hline 1 & S1-Sprache & so und denn $\sin =$ ma obn anjek' $O m m$. \\
\hline 2 & S1-Sprache & hh \\
\hline 3 & S1-Sprache & und denn hat hanne schlÜssel vergessn jehabt. \\
\hline 4 & S1-Sprache & $\mathrm{h}$ \\
\hline 5 & $\begin{array}{l}\text { S1-Sprache } \\
\text { S1-Geste } \\
\text { S1-Gestenform }\end{array}$ & $\begin{array}{l}\text { so und wie_n nu rin=in=i:n_ä:h inne wOHnung? } \\
\text { deiktisch } \\
\text { rH; Hf: flache Hand; Or: lateral, zum Zentrum; Bew: gerade nach } \\
\text { vorn; Pos: zentral, oben }\end{array}$ \\
\hline 6 & S1-Sprache & $\mathrm{h}$ \\
\hline 7 & \begin{tabular}{|l|} 
S1-Sprache \\
S1-Geste \\
S1-Gestenform
\end{tabular} & $\begin{array}{l}\text { es äh sones (.) wOhnhzimmerfensta stand ja off } \\
\text { metapragmatisch (PUOH) } \\
\text { rh; Hf: flache Hand; Or: oben, vom Körper weg; Bew: gerade nach } \\
\text { vorn; Pos: zentral, rechts oben }\end{array}$ \\
\hline 8 & S1-Sprache & hhh \\
\hline 9 & $\begin{array}{l}\text { S1-Sprache } \\
\text { S1-Geste }\end{array}$ & $\begin{array}{l}\text { tante gisel war d Aa nicht dabei= } \\
\text { Selbstberührung }\end{array}$ \\
\hline 10 & $\begin{array}{l}\text { S1-Sprache } \\
\text { S1-Geste } \\
\text { S1-Gestenform }\end{array}$ & $\begin{array}{l}\text { die sind denn } \\
\text { Beat } \\
\text { rh; Hf: flache Hand; Or: lateral, zum Körper; Bew: leicht gebogen, } \\
\text { nach vorn; Pos: zentral }\end{array}$ \\
\hline 11 & $\begin{array}{l}\text { S1-Sprache } \\
\text { S1-Geste } \\
\text { S1-Gestenform }\end{array}$ & $\begin{array}{l}\text { wahrscheinlich schon zu Euch jegang (unverständlich). } \\
\text { konkret referentiell + deiktisch (Weg+Verortung) } \\
\text { rh; Hf: flache Hand; Or: lateral, zum Körper; Bew: s-Linie, von rechts } \\
\text { nach links; Pos: zentral, links }\end{array}$ \\
\hline 12 & S2-Sprache & die hat noch wen gehOlt. \\
\hline 13 & \begin{tabular}{|l|} 
S1-Sprache \\
S1-Geste \\
S1-Gestenform
\end{tabular} & $\begin{array}{l}\text { oder ha_sind zurück ham wieder wen jeholt. } \\
\text { deiktisch (Richtungsangabe) } \\
\text { rh; Hf: flache Hand; Or: lateral, zum Köper und Zentrum ; Bew: gebo- } \\
\text { gen, nach links; Pos: peripher, rechts oben }\end{array}$ \\
\hline 14 & S1-Sprache & hhh \\
\hline 15 & \begin{tabular}{|l|} 
S1-Sprache \\
S1-Geste \\
S1-Gestenform
\end{tabular} & $\begin{array}{l}\text { und so jetzt wollte hanne da rinklettern=wir hattn ja all een zu sitzn } \\
\text { also nich stockbesoffen oder was. aber een zu sitzn. } \\
\text { Emblem } \\
\text { rh; Hf: flache Hand, Finger } 2 \text { bis } 5 \text { leicht gebeugt; Or: nach unten, zum } \\
\text { Körper; Bew: gerade; Pos: zentral, oben }\end{array}$ \\
\hline 16 & S1-Sprache & und Oma imma- \\
\hline 17 & S1-Sprache & $\mathrm{h}$ \\
\hline
\end{tabular}




\begin{tabular}{|c|c|c|}
\hline 18 & $\begin{array}{l}\text { S1-Sprache } \\
\text { S1-Geste } \\
\text { S1-Gestenform }\end{array}$ & $\begin{array}{l}\text { was WILLST DENN DU? } \\
\text { konkret referentiell (Schubsbewegung nach vorn) } \\
\text { bh; Hf: flache Hand; Or: vertikal, diagonal, vom Körper weg; Bew: ge- } \\
\text { rade nach vorn; Pos: zentral-zentral }\end{array}$ \\
\hline 19 & S1-Sprache & $\mathrm{h}$ \\
\hline 20 & $\begin{array}{l}\text { S1-Sprache } \\
\text { S1-Geste } \\
\text { S1-Gestenform }\end{array}$ & $\begin{array}{l}\text { GEH DU rUNta. } \\
\text { konkret referentiell (Schubsbewegung nach unten) } \\
\text { bh; Hf: flache Hand; Or: vertikal, diagonal, vom Körper weg; Bew: ge- } \\
\text { rade nach vorn und unten; Pos: zentral-zentral }\end{array}$ \\
\hline 21 & S1-Sprache & $\mathrm{h}$ \\
\hline 22 & $\begin{array}{l}\text { S1-Sprache } \\
\text { S1-Geste } \\
\text { S1-Gestenform }\end{array}$ & $\begin{array}{l}\text { weeßte dis war son ne } \\
\text { deiktisch } \\
\text { rh; Hf: einzelner Finger (2), Zeigefinger; Or: lateral, weg vom Körper; } \\
\text { Bew: gerade nach rechts; Pos: peripher, rechts }\end{array}$ \\
\hline 23 & $\begin{array}{l}\text { S1-Sprache } \\
\text { S1-Geste } \\
\text { S1-Gestenform }\end{array}$ & $\begin{array}{l}\text { zwEIraumwohnung hier mit som gItta. } \\
\text { deiktisch } \\
\text { lh; Hf: einzelner Finger (2), Zeigefinger; Or: lateral, weg vom Körper; } \\
\text { Bew: gerade nach links; Pos: peripher, links }\end{array}$ \\
\hline 24 & $\begin{array}{l}\text { S1-Sprache } \\
\text { S1-Geste } \\
\text { S1-Gestenform }\end{array}$ & $\begin{array}{l}\text { und hanne imma (.) Oma ich hab doch n schlÜssl vergessn= } \\
\text { rekurrent mit metapragmatischer (sehr lockere PUOH) als Teil der } \\
\text { Rollenübernahme } \\
\text { rh; Hf: flache Hand (leicht gebogen); Or: vertikal, weg vom Zentrum; } \\
\text { Bew: gerade, nach oben; Pos: peripher, links und rechts }\end{array}$ \\
\hline 25 & $\begin{array}{l}\text { S1-Sprache } \\
\text { S1-Geste } \\
\text { S1-Gestenform }\end{array}$ & $\begin{array}{l}\text { geh RUnta } \\
\text { konkret referentiell (Schubsbewegung nach vorn+unten) } \\
\text { bh; Hf: flache Hand; Or: vertikal, vom Körper weg; Bew: gebogen } \\
\text { vorn und unten; Pos: zentral }\end{array}$ \\
\hline 26 & S1-Sprache & (lacht) \\
\hline 27 & S1-Sprache & $\mathrm{h}$ \\
\hline 28 & $\begin{array}{l}\text { S1-Sprache } \\
\text { S1-Geste } \\
\text { S1-Gestenform }\end{array}$ & $\begin{array}{l}\text { und die hat Immer jeschubst- } \\
\text { konkret rerferentiell (Schubsbewegung nach vorn+unten) } \\
\text { bh; Hf: flache hand; Or: vertikal, vom Körper weg; Bew:gebogen vorn } \\
\text { und unten (1 Wiederholung); Pos: zentral }\end{array}$ \\
\hline 29 & $\begin{array}{l}\text { S1-Sprache } \\
\text { S1-Sprache } \\
\text { S1-Geste } \\
\text { S1-Gestenform } \\
\text { S3-Sprache } \\
\end{array}$ & $\begin{array}{l}\text { und wir hintn- } \\
(-) \\
\text { konkret referentiell (Drückbewegung nach oben) } \\
\text { bh; Hf: flache Hand; Or: vertikal, diagonal nach oben, weg vom Kö- } \\
\text { per; Bew: gerade nach oben; Pos: zentral, oben } \\
\text { Echt? }\end{array}$ \\
\hline 31 & $\begin{array}{l}\text { S2-Sprache } \\
\text { S1-Sprache }\end{array}$ & $\begin{array}{l}\text { (lacht) } \\
\text { ja. (lacht) }\end{array}$ \\
\hline
\end{tabular}




\begin{tabular}{|c|c|c|}
\hline 32 & $\begin{array}{l}\text { S1-Sprache } \\
\text { S1-Geste } \\
\text { S1-Gestenform }\end{array}$ & $\begin{array}{l}\text { wir immer hIntn HAnne wieder am arsche (.) } \\
\text { konkret referentiell (Drückbewegung nach oben) } \\
\text { bh; Hf: flache Hand; Or: vertikal, diagonal nach oben, weg vom Kö- } \\
\text { per; Bew: gerade nach oben (1 Wiederholung); Pos: zentral, oben }\end{array}$ \\
\hline 34 & $\begin{array}{l}\text { S1-Sprache } \\
\text { S1-Geste } \\
\text { S1-Gestenform }\end{array}$ & $\begin{array}{l}\text { HOch (.) } \\
\text { konkret referentiell (Drückbewegung nach oben) } \\
\text { bh; Hf: flache Hand; Or: vertikal, diagonal nach oben, weg vom Kö- } \\
\text { per; Bew: gerade nach oben; Pos: zentral, oben }\end{array}$ \\
\hline 35 & $\begin{array}{l}\text { S1-Sprache } \\
\text { S1-Geste } \\
\text { S1-Gestenform }\end{array}$ & $\begin{array}{l}\text { dass die (.) rInn. } \\
\text { konkret referentiell } \\
\text { rh; Hf: flache Hand, Finger } 2 \text { bis } 5 \text { leicht gebeugt; Or: vertikal, weg } \\
\text { vom Köper; Bew: gebogen nach unten; Pos: zentral, oben/peripher }\end{array}$ \\
\hline 36 & S1-Sprache & hh \\
\hline 37 & $\begin{array}{l}\text { S1-Sprache } \\
\text { S1-Geste } \\
\text { S1-Gestenform }\end{array}$ & $\begin{array}{l}\text { dis ging immer hIn und her `und gelacht. } \\
\text { pragmatisch (Abwinken) } \\
\text { rh; Hf. flache Hand, Finger } 2 \text { bis } 5 \text { loffen-gekrümmt; Or: vertikal, zum } \\
\text { Zentrum; Bew. gebogen; Pos: zentral, links oben }\end{array}$ \\
\hline 38 & $\begin{array}{l}\text { S1-Sprache } \\
\text { S1-Geste } \\
\text { S1-Gestenform }\end{array}$ & $\begin{array}{l}\text { 'wir hatten ja leicht een zu sitzen so=weeßte dis war so witzig=erste } \\
\text { mal witzig wEeßte? } \\
\text { Emblem } \\
\text { rh; Hf: flache Hand; Or: vertikal, zum Köper; Bew: gerade zum Körper } \\
\text { (2 Wiederholungen); Pos: zentral, oben rechts }\end{array}$ \\
\hline 39 & S1-Sprache & hier oma hat überhAupt nicht mitgekriegt was wir wOlln- \\
\hline 40 & $\begin{array}{l}\text { S1-Sprache } \\
\text { S1-Geste } \\
\text { S1-Gestenform }\end{array}$ & $\begin{array}{l}\text { h wir } \\
\text { metapragmatisch, PUOH } \\
\text { rh; Hf: flache Hand, Or: vertikal, weg vom Zentrum, weg vom Körper; } \\
\text { Bew: gerade nach oben, Pos: zentral, oben }\end{array}$ \\
\hline 41 & S1-Sprache & $\begin{array}{l}\text { wir hÄttn doch gar nich 'rEIn könn=wir hatt keen sch'lÜssel nüscht } \\
\text { 'wa. }\end{array}$ \\
\hline
\end{tabular}

\section{Anhang B: Legende zum Transkript}

\begin{tabular}{|l|l|}
\hline S-1, S-2, S-3 & Sprecherin 1, Sprecherin 2, Sprecherin 3 \\
\hline Hand & rechte Hand = rh, linke Hand = lh, beidhändig = bh \\
\hline Handform (Hf) & $\begin{array}{l}\text { Faust, flache Hand, einzelne Finger, Kombinationen von Fingern (1 = } \\
\text { Daumen, 2 = Zeigefinger, 3 = Mittelfinger, 4 = Ringfinger, 5 = klei- } \\
\text { ner Finger) }\end{array}$ \\
\hline $\begin{array}{l}\text { Orientierung der } \\
\text { Handfläche (Or): }\end{array}$ & $\begin{array}{l}\text { oben, unten, lateral, vertikal; zum/weg vom Zenter, zum Körper/weg } \\
\text { vom Körper }\end{array}$ \\
\hline Bewegung (Bew): & gerade, gebogen, kreisförmig, zick-zack, s-Linie, spiralförmig \\
\hline
\end{tabular}




\begin{tabular}{|l|l|}
\hline $\begin{array}{l}\text { Position im Gesten- } \\
\text { raum (Pos): }\end{array}$ & zentral-zentral, zentral, peripher; links, rechts, oben, unten \\
\hline $\begin{array}{l}\text { und wie_n nu } \\
\text { rin=in=i:n }\end{array}$ & Fettdruck: stroke phase (bedeutungstragende Phase) der Geste \\
\hline & Phase der Klimax \\
\hline & Klimax \\
\hline Gestennotation via Formeigenschaften nach Bressem (2013) \\
\hline Gestenraum kodiert nach McNeill (1992: 89). \\
\hline
\end{tabular}

